\title{
Computational Techniques for Parameter Estimation of Gravitational Wave Signals
}

\author{
Renate Meyer ${ }^{1}$, Matthew C. Edwards ${ }^{1}$, Patricio Maturana-Russel ${ }^{1,2}$, and Nelson \\ Christensen ${ }^{3}$ \\ ${ }^{1}$ Department of Statistics, The University of Auckland, Auckland, New Zealand \\ ${ }^{2}$ Department of Mathematical Sciences, Auckland University of Technology, \\ Auckland, New Zealand \\ ${ }^{3}$ Artemis, Observatoire de la Côte d'Azur, Université Côte d'Azur, Nice, France
}

\begin{abstract}
Since the very first detection of gravitational waves from the coalescence of two black holes in 2015, Bayesian statistical methods have been routinely applied by LIGO and Virgo to extract the signal out of noisy interferometric measurements, obtain point estimates of the physical parameters responsible for producing the signal, and rigorously quantify their uncertainties. Different computational techniques have been devised depending on the source of the gravitational radiation and the gravitational waveform model used. Prominent sources of gravitational waves are binary black hole or neutron star mergers, the only objects that have been observed by detectors to date. But also gravitational waves from core collapse supernovae, rapidly rotating neutron stars, and the stochastic gravitational wave background are in the sensitivity band of the ground-based interferometers and expected to be observable in future observation runs. As nonlinearities of the complex waveforms and the high-dimensional parameter spaces preclude analytic evaluation of the posterior distribution, posterior inference for all these sources relies on computer-intensive simulation techniques such as Markov chain Monte Carlo methods. A review of state-of-the-art Bayesian statistical parameter estimation methods will be given for researchers in this cross-disciplinary area of gravitational wave data analysis.
\end{abstract}

This is the peer reviewed version of the following article: "Computational Techniques for Parameter Estimation of Gravitational Wave Signals", which has been published in final form at http://dx.doi.org/10.1002/wics.1532. This article may be used for non-commercial purposes in accordance with Wiley Terms and Conditions for Use of Self-Archived Versions. 


\section{Introduction}

The era of observational gravitational wave astronomy truly began with the detection of GW150914 - gravitational waves from the inspiral and merger of two stellar-mass black holes that coalesced to form a single rotating black hole - by the two Advanced Laser Interferometer Gravitational-wave Observatory (Advanced LIGO) detectors (B. Abbott et al., 2016a) in 2015. Even though the existence of gravitational waves had already been predicted by Einstein in 1916 (Einstein, 1916) as a consequence of the theory of General Relativity, only indirect evidence had so far been provided by radio observations of the binary pulsar PSR1913+16 in 1974 and the double pulsar PSR J0737-3039 in 2003. Taylor $\&$ Weisberg (1982) showed that the energy loss associated with the orbital decay rate was consistent with the emission of gravitational waves. The discoverers of PSR1913+16 (Hulse \& Taylor, 1975), Joseph Taylor and Russell Hulse, were awarded the Nobel Prize in Physics in 1993. The groundbreaking first direct measurement of gravitational waves earned the founders of LIGO, Rainer Weiss, Barry Barish, and Kip Thorne, the Nobel Prize in Physics in 2017. Since then, many more black hole mergers have been observed in subsequent observation runs of the Advanced LIGO and Virgo interferometers (B. Abbott et al., 2019d; R. Abbott et al., 2020; B. Abbott et al., 2020b,c). The detection of the neutron star merger GW170817 (B. Abbott et al., 2017c), seen both in gravitational and electromagnetic waves, ushered in the new era of gravitational wave multi-messenger astronomy (B. Abbott et al., 2017e).

Gravitational waves are produced by non-axisymmetric acceleration of mass, such as two compact neutron stars orbiting each other. They are quadrupole (lowest order) waves that propagate outwards from their source at the speed of light. Unlike electromagnetic waves, they pass through any matter. Their effect is orthogonal to the direction of propagation. They have two polarizations, a plus- and cross- polarization. The plus-polarization stretches the distance of two points on the horizontal axis and simultaneously compresses the distance between two points on the vertical axis. The cross-polarization has a similar effect, but rotated by 45 degrees. Gravitational wave detectors, such as Advanced LIGO (Aasi et al., 2015) and Advanced Virgo (Acernese et al., 2015), are based on Michelson laser interferometry (Hariharan, 2007; Pitkin et al., 2011). A beam of light is split in two and sent in two equal-length (4 km for LIGO, $3 \mathrm{~km}$ for Virgo) perpendicular arms (in vacuum) with mirror-coated test masses suspended on pendulums at each end of the arms, storing the light and increasing the effective arm length by a factor of $\sim 300$ as the light bounces back and forth. The light beams exiting the arms are recombined. A passing gravitational wave changes the lengths of each arm and thus the interference pattern measured by photodetectors. The wave amplitude, the dimensionless strain, denoted by $h$, is measured by the relative change in spacing $\frac{\Delta L}{L}$ between two test masses where $L$ denotes the equilibrium spacing. The strain is proportional to the second time derivative of the source quadrupole moment and decreases in proportion to the inverse distance from the source (Schutz, 2009). Thus, in practice only gravitational waves from massive and rapidly accelerating objects in the Universe will be detectable. Gravitational waves from astronomical sources, due to 
their large distance from Earth, have detectable strains of the order of $10^{-21}$.

Here, we focus on observations from the network of ground-based interferometers. After a five-year long upgrade, Advanced LIGO became operational in 2015 with two detectors, in Hanford, Washington and Livingston, Louisiana, and Advanced Virgo with a detector in Cascina, Italy, in 2017. A third LIGO detector is planned to be built in India (Unnikrishnan, 2013), and the Japanese underground cryogenic detector KAGRA (Somiya, 2012) is currently coming online with its commissioning activities. With a single detector, it is difficult to determine the sky location of a transient source. A world-wide network of interferometers is important to estimate the source position using timing, amplitude, and polarization information of the signal (Bizouard \& Papa, 2013). The Einstein Telescope (Sathyaprakash et al., 2012; Maggiore et al., 2020) is a proposed third generation underground cryogenic detector with $10 \mathrm{~km}$ arm lengths in a triangular formation. The Cosmic Explorer, a planned $40 \mathrm{~km} \mathrm{~L}$-shaped detector, will greatly enhance the sensitivity due to the significantly increased arm lengths (B. Abbott et al., 2017a). Pulsar timing arrays (Dahal, 2020) are sensitive to low-frequency gravitational waves in the range of $10^{-9}$ to $10^{-6} \mathrm{~Hz}$. Furthermore, a space-based interferometer, the so-called Laser Interferometer Space Antenna (LISA) is planned to be launched in 2034 by the European Space Agency with three satellites 2.5 million $\mathrm{km}$ apart, forming an equilateral triangle in an Earth-trailing, heliocentric orbit (Amaro-Seoane et al., 2017). A space-based interferometer would be sensitive to frequencies from $0.1 \mathrm{mHz}$ to $1 \mathrm{~Hz}$. Because LIGO and Virgo are currently operating, and making detections, this review is concentrating on parameter estimation methods for these ground based detectors. However the parameter estimation development for LISA has been long and active (Umstätter et al., 2005a,b; N. J. Cornish \& Crowder, 2005; Crowder \& Cornish, 2007; Babak et al., 2008; Stroeer et al., 2007; Arnaud et al., 2007; Ali et al., 2012; Baghi et al., 2019; Katz et al., 2020; Toubiana et al., 2020). LISA will actually observe thousands of simultaneous signals, and therefore pose one of the biggest parameter estimation challenge ever in physics (Babak, 2017; Robson \& Cornish, 2017; N. Cornish \& Robson, 2017; T. Littenberg et al., 2020; Marsat et al., 2020; N. Cornish \& Shuman, 2020).

Even though much information about the emitting source of the gravitational waves can be learned from the direct inspection of the observed gravitational waveforms (LIGO Scientific \& Virgo Collaborations, 2017), the full posterior distribution of the source parameters using Bayesian computational techniques is required to accurately estimate the properties of the source, such as the masses and spins of the two black holes, and to quantify associated uncertainties. A review of state-of-the-art parameter estimation methods will be the focus of this paper. As this is not only an expansive but also a rapidly evolving research area, we can only strive for a comprehensive review but cannot claim exhaustiveness. For details regarding the computational methodology and their implementations, we refer to the relevant statistical and machine learning literature. 


\section{Parameter Estimation}

Following a calibration procedure for each detector, the time series of dimensionless strain measurements are produced. The strain is the fractional change in spacings between two test masses due to a passing gravitational wave, that is the difference in lengths relative to the total arm length of the interferometer. The calibrated observations $\mathbf{d}^{(k)}=\left(d^{(k)}(t)\right), t=$ $1, \ldots, T$ of gravitational waves from detector $k$ are modelled as a deterministic signal, the strain $\mathbf{h}^{(k)}=\left(h^{(k)}(t \mid \boldsymbol{\theta})\right), t=1, \ldots, T$ plus additive interferometer noise $n^{(k)}(t)$, that is,

$$
d^{(k)}(t)=h^{(k)}(t \mid \boldsymbol{\theta})+n^{(k)}(t), \quad t=1, \ldots, T .
$$

Thus, the model consists of two parts: a model for the gravitational wave signal and a model for the noise, both equally important for parameter estimation (B. Abbott et al., 2020a).

The interferometers are subject to a variety of noise components including quantum noise, seismic noise, thermal noise, and gravity gradient noise. Also, environmental noise or malfunctioning of equipment can cause transient noise events (B. Abbott et al., 2016; Nuttall, 2018), or noise spectral lines (Covas et al., 2018). All these combined are modelled by the noise time series $n^{(k)}(t)$, usually assumed to be a Gaussian, stationary time series with zero mean and covariance matrix $\Sigma_{k}$.

The assumptions on the noise determine the form of the likelihood. The observations from different detectors in a network of $K$ detectors are usually assumed to be independent and thus, for a coherent analysis that includes data from all detectors, the joint likelihood is the product of the individual likelihood functions:

$$
L(\mathbf{d} \mid \boldsymbol{\theta})=\prod_{k=1}^{K} L^{(k)}\left(\mathbf{d}^{(k)} \mid \boldsymbol{\theta}\right)=\prod_{k=1}^{K} \frac{1}{\operatorname{det}\left(2 \pi \Sigma_{k}\right)^{1 / 2}} \mathrm{e}^{-\frac{1}{2}\left(\mathbf{d}^{(k)}-\mathbf{h}^{(k)}\right)^{\top} \Sigma_{k}^{-1}\left(\mathbf{d}^{(k)}-\mathbf{h}^{(k)}\right)}
$$

Usually, parameter estimation is implemented in the frequency domain because after a Fourier transform, instead of a multivariate Gaussian likelihood with non-diagonal covariance matrix, the complex vector $\tilde{\mathbf{d}}^{(k)}$ containing Fourier coefficients defined by

$$
\tilde{d}_{j}^{(k)}=\tilde{d}^{(k)}\left(f_{j}\right)=\sum_{t=1}^{T} d^{(k)}(t) \mathrm{e}^{-i t f_{j}}
$$

at the Fourier frequencies $f_{j}=2 \pi j / T, j=0, \ldots, N, N=\lfloor(T-1) / 2\rfloor$, is approximately (for large $T$ ) a complex multivariate Gaussian but with a diagonal covariance matrix $S^{(k)}$ that contains the power spectral density $S^{(k)}\left(f_{j}\right)$ at the Fourier frequencies $f_{j}$ on the diagonal. The power spectral density

$$
S^{(k)}(f)=\sum_{\ell=-\infty}^{\infty} \gamma^{(k)}(\ell) \mathrm{e}^{-i \ell f}
$$


is the Fourier transform of the autocovariance function $\gamma^{(k)}(l)$ of the stationary noise time series. The diagonal covariance structure in this so-called Whittle likelihood approximation (Whittle, 1957; Finn, 1992; Cutler \& Flanagan, 1994; Kirch et al., 2019) facilitates the calculation of the inverse and determinant in the Whittle likelihood:

$$
L(\mathbf{d} \mid \boldsymbol{\theta}) \approx \prod_{k=1}^{K} \frac{1}{\operatorname{det}\left(\pi T \mathbf{S}^{(k)}\right)} \mathrm{e}^{-\frac{1}{T}\left(\tilde{\mathbf{d}}^{(k)}-\tilde{\mathbf{h}}^{(k)}\right)^{*} \mathbf{S}^{(k)^{-1}}\left(\tilde{\mathbf{d}}^{(k)}-\tilde{\mathbf{h}}^{(k)}\right)}
$$

In the following, we assume that the power spectral density (PSD) for each detector is known but in Section 7 we outline procedures to deal with an unknown noise spectral density that is estimated simultaneously with the signal parameters. The stationarity assumption is usually adequate for transient signals such as the signal from a merger of two black holes but it is well known that the interferometer noise is slowly time-varying over longer periods of about one minute (Chatziioannou et al., 2019). Therefore, it will be important to take the time-varying noise into account when estimating the parameters of gravitational wave signals of longer duration such as those produced by neutron star mergers or pulsars. Furthermore, the power spectrum contains many high power narrow spectral lines which are not compatible with the Gaussian assumption. Many of these are due to known sources such as the $60 \mathrm{~Hz}$ power line harmonics, so-called 'violin modes' caused by thermally excited mirror suspension and their harmonics, or calibration lines inserted by moving the end mirrors (Covas et al., 2018). Berry et al. (2015) perform a systematic study on the performance of parameter estimates under the Gaussian assumption but with real, non-ideal noise. Figure 1 shows the amplitude spectral density (the square root of the power spectral density) of both Advanced LIGO detectors and the Advanced Virgo detector from their second observational run (B. Abbott et al., 2019d). For the purpose of parameter estimation, the power spectral density is usually assumed to be known and fixed. Its value is usually obtained by one of two methods. The Welch method (Welch, 1967) uses a separate stretch of data close to but not containing the time period of the signal. This stretch of data is divided into overlapping segments of the same duration $T$ as the signal period. These segments are Fourier transformed using the FFT algorithm after windowing with a Tukey or Hanning window to avoid spectral leakage (B. Abbott et al., 2020a). The Welch method averages over the periodograms of each individual time segment to reduce the variance of the estimate. Often, the median instead of mean of the periodograms is used as it is more robust with respect to outliers (Veitch et al., 2015). As an alternative to this off-source Welch method, an on-source estimate, based on the same data that contains the signal, is obtained by BayesWave (N. J. Cornish \& Littenberg, 2015), described in Section 7. The power spectral density is then assumed to be known and equal to the Welch or BayesWave estimate when estimating the parameters of the signal. The likelihood Eq. (4) for known PSD simplifies to

$$
L(\mathbf{d} \mid \boldsymbol{\theta}) \propto \prod_{k=1}^{K} \mathrm{e}^{-\frac{1}{T}\left(\tilde{\mathbf{d}}^{(k)}-\tilde{\mathbf{h}}^{(k)}\right)^{*} \mathbf{S}^{(k)-1}\left(\tilde{\mathbf{d}}^{(k)}-\tilde{\mathbf{h}}^{(k)}\right)}
$$




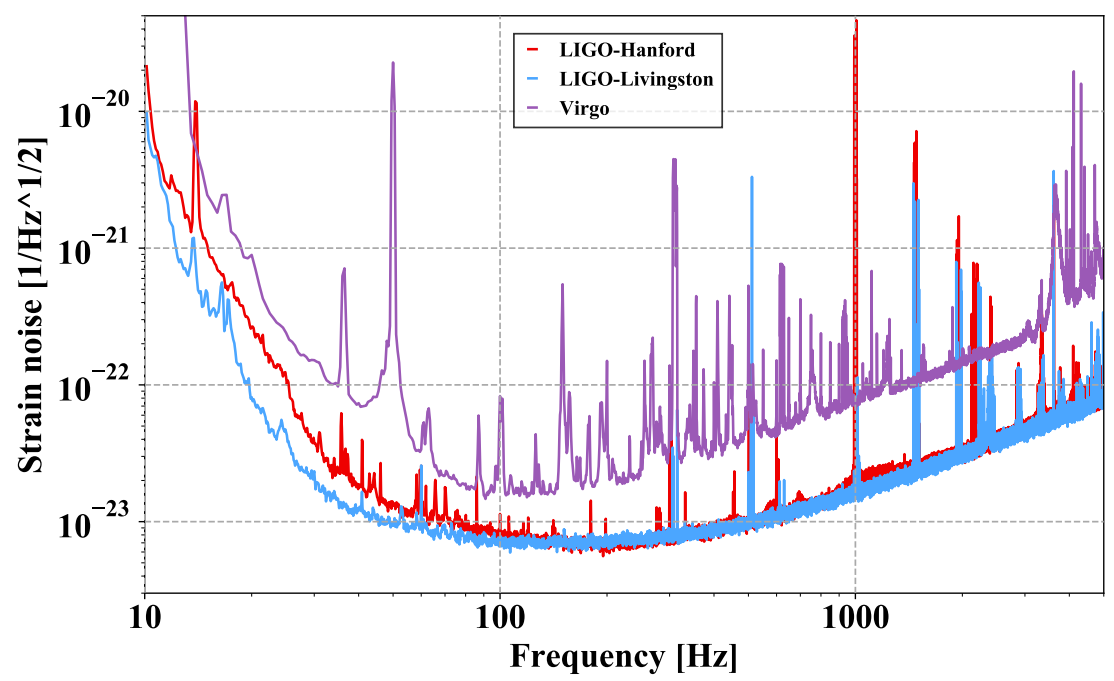

Figure 1: The Advanced LIGO and Advanced Virgo sensitivity curves. These are typical of the best sensitivities of the detectors during their second observational run (B. Abbott et al., 2019d). Source: LIGO Scientific Collaboration

Alternative parametric and nonparametric Bayesian estimates of the power spectral density are discussed in Section 7.

The strain signal is characterized by various parameters (depending on the gravitational wave source) such as the masses and spins of the progenitors in case of a compact binary coalescence, compiled in the parameter vector $\boldsymbol{\theta}=\left(\theta_{1}, \ldots, \theta_{p}\right)$. Considering a geocentric reference frame, the strain measured at detector $k$ of a gravitational wave source with polarization amplitudes $h_{+}$and $h_{\times}$located in the sky at $(\alpha, \delta)$ where $\alpha$ is the right ascension and $\delta$ the declination of the source is

$$
h^{(k)}(t \mid \boldsymbol{\theta})=F_{+}^{(k)}(\alpha, \delta, \psi) h_{+}\left(t-\tau^{(k)} \mid \boldsymbol{\theta}\right)+F_{\times}^{(k)}(\alpha, \delta, \psi) h_{\times}\left(t-\tau^{(k)} \mid \boldsymbol{\theta}\right) .
$$

$F_{+, \times}$are the antenna response functions that depend on the source locations, the polarization angle $\psi$ of the waves (Christensen, 1992; Anderson et al., 2001), plus the locations and orientations of the detectors. For short transient signals, the time dependence of the antenna response functions due to the rotation of the earth can be safely ignored and assumed to be constant throughout the observation period, but needs to be taken into account for long signals. The parameter $\tau^{(k)}=\tau^{(k)}(\alpha, \delta)$ denotes the location-dependent time delay. For a detailed explanation of the calibration of the gravitational wave detectors and methods to take the associated calibration uncertainty into account, we refer to Sun et al. (2020); Viets et al. (2018); Cahillane et al. (2017); Acernese et al. (2018).

Some pre-processing steps are necessary. The time series is usually down sampled from its 
original sampling frequency of $16384 \mathrm{~Hz}$ for LIGO and $20 \mathrm{kHz}$ for Virgo to a lower rate (typically $4096 \mathrm{~Hz}$ ). Then it is band-pass filtered because the LIGO detectors are calibrated only in the frequency band from $10 \mathrm{~Hz}$ to $5 \mathrm{kHz}$ and the Virgo detector is calibrated in the band from $10 \mathrm{~Hz}$ to $8 \mathrm{kHz}$. In some analyses, the time series is also notch filtered around known instrumental noise frequencies. Software packages for pre-processing are included in the LAL library (LIGO Scientific Collaboration, 2018) and the Gravitational Wave Open Science Center (R. Abbott et al., 2019; LIGO Scientific Collaboration, Virgo Collaboration, 2020b). Parameter estimation is often performed in the frequency domain. After a discrete Fourier transform, model (1) is equivalent to the following frequency domain model:

$$
\tilde{d}_{j}=\tilde{d}\left(f_{j}\right)=\tilde{h}^{(k)}\left(f_{j} \mid \boldsymbol{\theta}\right)+\tilde{n}^{(k)}\left(f_{j}\right), \quad f_{j}=2 \pi j / T, \quad j=0, \ldots, N
$$

with

$$
\tilde{h}^{(k)}\left(f_{j} \mid \boldsymbol{\theta}\right)=\left(F_{+}^{(k)}(\alpha, \delta, \psi) \tilde{h}_{+}\left(f_{j} \mid \boldsymbol{\theta}\right)+F_{\times}^{(k)}(\alpha, \delta, \psi) \tilde{h}_{\times}\left(f_{j} \mid \boldsymbol{\theta}\right)\right) \mathrm{e}^{-i f_{j} \tau^{(k)}}
$$

The exact form of the gravitational waveform model $h_{+, \times}(t \mid \boldsymbol{\theta})$ depends on the emitting source of the gravitational waves. In the following sections, we describe these waveform models from compact binary coalescences, burst signals from core-collapse supernovae, continuous signals from rapidly rotating neutron stars (pulsars), and stochastic signals from astrophysical and cosmological origins that combine to form the stochastic gravitational wave background, illustrated in Figure 2. For each of these gravitational waveform sources, we will specify the computational techniques used to estimate their parameters. In Section 7 we describe methods for estimating the unknown noise spectral density in unison with the signal parameters. 

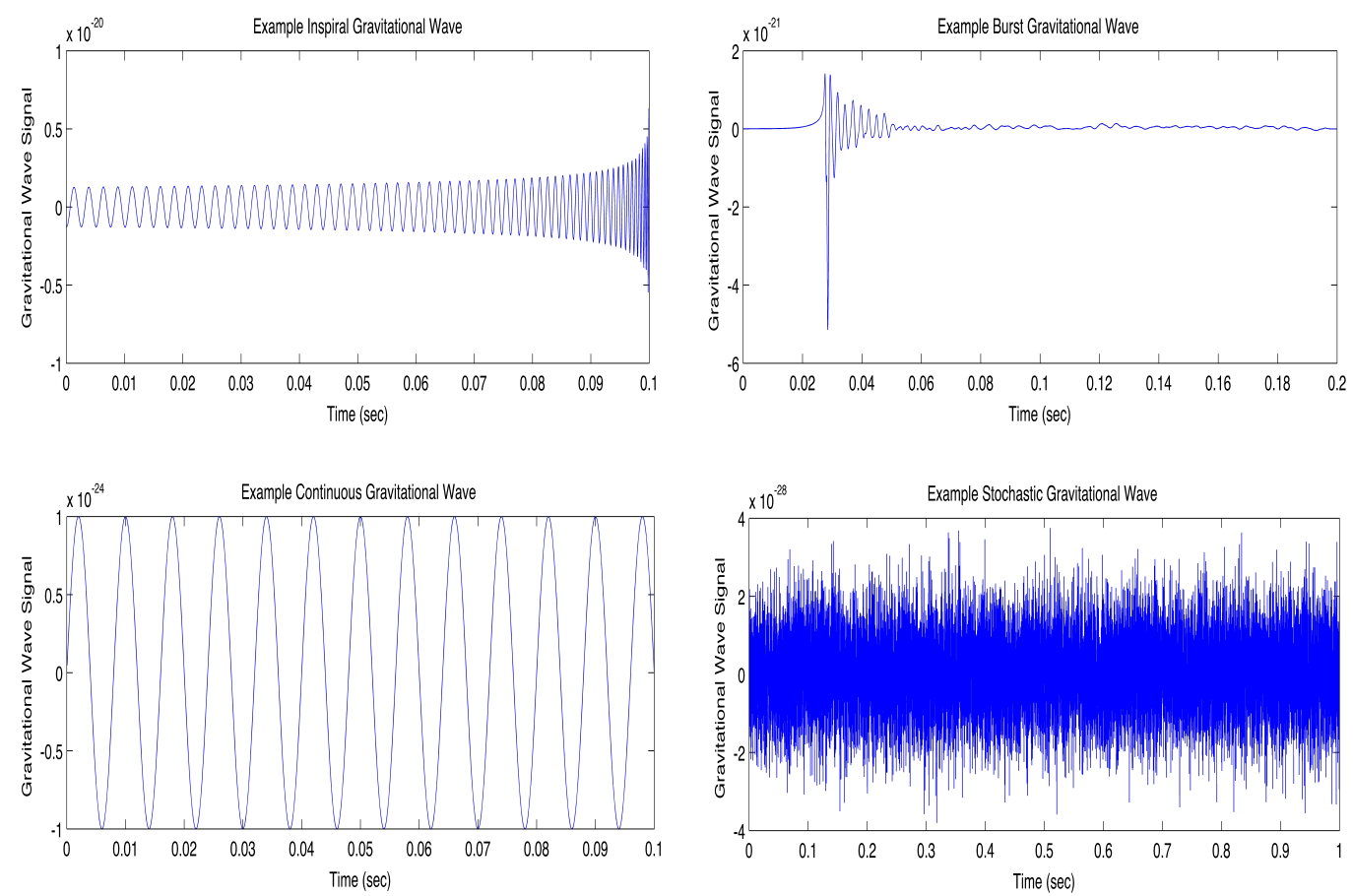

Figure 2: Simulated gravitational wave signals with different waveforms: a compact binary inspiral, core-collapse supernova, continuous wave, and stochastic gravitational wave signal. Credit: A. Stuver, LIGO Scientific Collaboration (Stuver, 2020). 


\section{Compact Binary Coalescences}

To date, the Advanced LIGO and Advanced Virgo detectors have observed gravitational waves from the coalescence of dozens of compact binary systems containing black holes and also two neutron star mergers. Chirp-like signals of short duration, such as GW150914 (B. Abbott et al., 2016c), are generated during the final stage of a binary system as the two progenitor masses spiral in toward one another and then merge. Many different waveform families for binary mergers exist in the literature. Most are parametric waveform models obtained by solving Einstein's equations and can be constructed within different frameworks. They can be based on solving the two-body dynamics in general relativity perturbatively using post-Newtonian (PN) approximation of various orders (Buonanno et al., 2009; Blanchet, 2014; Damour, 2016). Two other frameworks were considered for the analyis of GW150914: the effective-one-body (EOBNR) waveforms (Buonanno \& Damour, 1999; Pürrer, 2016; Nagar et al., 2018; Ossokine et al., 2020) where higher order PN terms are calibrated to numerical relativity (NR) simulations, and hypbrid/phenomenological (IMRPhenom) waveforms (Hannam et al., 2014; Khan et al., 2016; Pratten et al., 2020) based on extending frequency-domain PN expressions and hybridizing PN and EOB with NR waveforms. For the simulation of gravitational wave signals, numerical relativity calculations have become very important (Brügmann, 2018; Eisenstein, 2019). Surrogate models of NR waveforms have been shown to be both fast and accurate (Varma et al., 2019).

When dealing with a binary neutron star system, or a neutron star - black hole binary, the neutron star can be distorted from its spherical shape from the tidal gravitational fields that it experiences. This deformation will actually increase the rate of the inspiral. In this case the gravitational waveform model should address this deformation. For example, with the examination of the gravitational wave data from the binary neutron star merger GW170817 (B. Abbott et al., 2017c) the waveform used was generated with the postNewtonian and EOBNR formalisms, which also included tidal deformations (Vines et al., 2011; B. Abbott et al., 2019f).

Here we exemplify the parameter estimation using $8 \mathrm{~s}$ of data around the transient event GW150914 (B. Abbott et al., 2016d; Romero-Shaw et al., 2020) from both Advanced LIGO detectors, comprising about ten cycles during the inspiral phase, followed by merger and ring-down. Merging black holes in a quasi-circular orbit are described by eight intrinsic parameters, the masses $m_{1,2}$ and the spins $s_{1,2}$ (in terms of the dimensionless spin magnitude $a=c|s| /\left(G m^{2}\right) \in[0,1]$ and orientation) of the individual black holes and an additional seven extrinsic parameters, the luminosity distance $D_{L}$, the right ascension $\alpha$ and declination $\delta$ of the source, the orientation in terms of the inclination angle $\iota$ between the system's orbital angular momentum and the line of sight, and the polarization angle $\psi$, the time $t_{c}$ and the phase $\phi_{c}$ of coalescence. If the spins of the masses are parallel to the orbital plane, then there can be a spin-orbit coupling that will cause a precession of the orbital plane. In this case $\iota$ is not well defined, and it is more appropriate to define the angle between the total angular momentum of the system and the line of sight, $\theta_{J N}$. We will ignore orbital 
precession for the remainder of this paper and continue to use $\iota$. For GW150914, the orbital eccentricity of the binary system was not considered. This would have added an additional two parameters, the magnitude and the argument of periapsis of the system.

During the inspiral phase, the gravitational wave polarizations observed at the angle $\iota$ can be expressed at the leading order as

$$
\begin{aligned}
& h_{+}(t \mid \boldsymbol{\theta})=A(t \mid \boldsymbol{\theta}) \frac{1}{2}\left(1+\cos ^{2} \iota\right) \cos \phi(t \mid \boldsymbol{\theta}) \\
& h_{\times}(t \mid \boldsymbol{\theta})=A(t \mid \boldsymbol{\theta}) \cos \iota \sin \phi(t \mid \boldsymbol{\theta})
\end{aligned}
$$

where $A(t \mid \boldsymbol{\theta})$ and $\phi(t \mid \boldsymbol{\theta})$ are the gravitational wave amplitude and phase, respectively. The gravitational wave frequency $f$ equals twice the orbital frequency. Due to the emission of gravitational waves the binary system loses energy causing the orbital distance to decrease and the orbital frequency to increase. The phase evolution $\phi(t)$ in the inspiral regime is well described by post-Newtonian theory, a perturbative expansion in powers of the orbital velocity $v / c$. The first order gravitational wave frequency evolution is described by the differential equation (LIGO Scientific \& Virgo Collaborations, 2017)

$$
\frac{d f}{d t}=\frac{96}{5} \pi^{8 / 3}\left(\frac{G \mathcal{M}}{c^{3}}\right)^{5 / 3} f^{11 / 3}
$$

where $c$ is the speed of light and $G$ Newton's gravitational constant. We see that the phase evolution is mainly influenced by the chirp mass $\mathcal{M}=\frac{\left(m_{1} m_{2}\right)^{3 / 5}}{\left(m_{1}+m_{2}\right)^{1 / 5}}$ as a result of energy loss from emitting gravitational waves. The chirp mass can be much more accurately estimated than the individual masses. Additional parameters such as the mass ratio $q=m_{1} / m_{2}$ (or equivalently the symmetric mass ratio $\left.\eta=\frac{m_{1} m_{2}}{\left(m_{1}+m_{2}\right)^{2}}=\frac{q}{(1+q)^{2}}\right)$ and the spin components enter at each of the following PN orders. This is accurate in the inspiral phase but degrades as the black holes get closer and eventually the full solution to Einstein's equations is needed using numeral relativity. Merger and ringdown depend primarily on the mass and spin of the final black hole. Note that the observed frequency of the signal is redshifted by a factor $(1+z)$ where $z$ is the cosmological redshift (Krolak \& Schutz, 1987) and cannot be distinguished from a rescaling of the masses by the same factor. Thus the source mass is obtained by dividing the measured redshifted mass by $(1+z)$. When the luminosity distance is estimated this can be converted to a redshift $z$ by assuming a Lambda Cold Dark Matter $(\Lambda C D M)$ cosmology and an appropriate value of the Hubble constant (Ade et al., 2016).

After a Fourier transform of the strain time series, parameter estimation is performed in the frequency domain using the likelihood function (4). Note that most waveform models used in practice are constructed in the frequency domain. That includes phenomenological models, surrogate models of effective-one-body waveforms and surrogate models built directly from NR waveforms. The Bayesian model is completed by specifying prior distributions for the parameters. For parameter estimation of GW150914, the masses were assumed to have a uniform prior on $[10,80] \mathbf{M}_{\odot}$ with $m_{2} \leq m_{1}$. The spin magnitudes $a_{1,2}$ were assumed to 
be uniformly distributed on $(0,1)$ and the spin orientations isotropic on the 2-sphere. The prior on $t_{c}$ was chosen to be uniform, centered at the reported time of coalescence with a width of $0.2 \mathrm{~s}, \phi_{c}$ and $\psi$ were assumed to be uniform on $[0,2 \pi]$. As the density of sources is assumed uniform in the cosmological co-moving volume, the prior for the source location in the Universe was isotropic, i.e. the prior for $\alpha$ uniform on $[0,2 \pi]$ and the prior for $\cos (\delta)$ uniform on $[0,1]$, and the distance prior is uniform in Euclidian volume. The prior on the cosine of the inclination angle $\iota$ was uniform on $[0,1]$.

The product of prior and likelihood determines the posterior distribution of the parameters using Bayes' theorem

$$
p(\boldsymbol{\theta} \mid \mathbf{d})=\frac{L(\mathbf{d} \mid \boldsymbol{\theta}) p(\boldsymbol{\theta})}{\int L(\mathbf{d} \mid \boldsymbol{\theta}) p(\boldsymbol{\theta}) d \boldsymbol{\theta}}
$$

A comprehensive treatment of Bayesian inference can be found in the textbook of Gelman et al. (2014) and an introduction to Bayesian inference for astronomers in Thrane \& Talbot (2019). Evaluation of the normalizing constant in the denominator (also called the marginal likelihood or evidence) and calculating marginal distributions and their summary statistics of individual parameters requires high-dimensional integration. To solve these high-dimensional integration problems, computer-intensive simulation-based methods are required for several reasons: the posterior distribution is not tractable analytically, numerical integration is only feasible in low dimensions, Laplace approximation (Gelman et al., 2014) is suitable only for unimodal and symmetric posteriors, and ordinary simulation methods based on independent random draws such as importance sampling (Gelman et al., 2014) are also only applicable effectively in low dimensions. Christensen \& Meyer (1998) demonstrated the use of Markov chain Monte Carlo (MCMC) methods for gravitational wave parameter estimation for posterior computation using a simple low-order waveform model with four parameters. MCMC methods were readily taken up by LIGO - Virgo and increasingly sophisticated MCMC techniques were developed to handle higher order waveform approximations with increasing number of parameters (Christensen \& Meyer, 2001; Pai et al., 2001; Christensen, Meyer, \& Libson, 2004; Röver et al., 2006; Röver et al., 2007; van der Sluys, Röver, et al., 2008; van der Sluys, Raymond, et al., 2008; Veitch \& Vecchio, 2010; Aasi et al., 2013). Implemented in LALInference is an adaptive version of the Metropolis-Hastings algorithm (Metropolis et al., 1953) coupled with parallel tempering (Swendsen \& Wang, 1986; Veitch et al., 2015) which is sufficiently flexible to handle any of the waveform models in the LAL libraries. For a detailed description of the parallel tempering MH algorithm, see van der Sluys, Röver, et al. (2008); van der Sluys, Raymond, et al. (2008).

Parallel tempering uses a series of functional probability densities also known as power posterior densities, which generate a bridge between the prior and the posterior distributions. The sampling is performed on these bridging densities, but allowing point swaps in adjacent chains, according to a certain probability. Thus, the exploration of the posterior distribution is prevented from being stuck in certain areas of the parameter space. This algorithm has been implemented in software packages such as LALinference (Veitch et al., 
2015) and BayesWave (N. J. Cornish \& Littenberg, 2015). Even though the samples from these intermediate bridging densities are discarded from the parameter inference process, these samples can be used to accurately calculate the marginal likelihood (e.g., T. B. Littenberg \& Cornish, 2009; Veitch et al., 2015; Maturana-Russel, Meyer, et al., 2019). They can also be used to build a proposal distribution based upon estimation of the kernel density and tuned to the target posterior (Farr et al., 2014). The open-source Python-based parameter estimation toolkit for compact binary coalescence signals, PyCBC Inference (Biwer et al., 2019), uses the ensemble MCMC algorithm emcee (Foreman-Mackey et al., 2013) and its parallel-tempered version emcee_pt (Vousden et al., 2015).

An alternative algorithm, also implemented in LALInference and routinely used together with the MCMC algorithm is nested sampling (NS) (Skilling, 2006), which evaluates the evidence/marginal likelihood, i.e. the denominator in Bayes theorem (Eq. 12). It can also be extended to generate samples from the posterior distribution at no extra cost. Its particular way of exploring the parameter space allows it to work in cases in which popular MCMC methods fail (Maturana-Russel, Brewer, et al., 2019). Basically, it does this by sampling a number of points from the prior and then the one with the lowest likelihood value is replaced by a new point drawn from the prior, but restricted to have a likelihood higher than the one that is being replaced. This procedure is repeated multiple times, allowing to keep points in different modes simultaneously and deal with complex likelihood functions. NS was first used for gravitational wave searches with ground-based observatories (Veitch \& Vecchio, 2008b,a). As it can generate samples from the posterior, it was then applied for parameter estimation and model selection for binary inspiral systems (Veitch \& Vecchio, 2010). NS type algorithms have also been used for model selection and parameter estimation of space-based detectors (e.g., Gair \& Porter, 2009; Feroz et al., 2009; Gair et al., 2010; Marsat et al., 2020). NS is available in computational packages such as Bilby (Ashton et al., 2019; Romero-Shaw et al., 2020) (a flexible Python-based package that also includes several MCMC samplers) and in parallelised versions (R. Smith \& Ashton, 2019), which can be used in computing clusters.

Both MCMC and NS were used to estimate the parameters of the very first gravitational wave signal GW150914 observed by Advanced LIGO, yielding consistent sets of parameter estimates (B. Abbott et al., 2016d). A reconstruction of the gravitational wave signal is shown in Figure 3. A table with parameter estimates and their standard errors can be found in Table I of B. Abbott et al. (2016d). The importance that Bayesian parameter estimation played in describing the physics associated with the first direct observation of gravitational waves with GW150914 is summarized in Meyer \& Christensen (2016).

In a compact binary coalescence event that includes at least one neutron star - a binary neutron star or a neutron star - black hole merger - electromagnetic signatures at different timescales and wavelengths are expected if the neutron star is tidally disrupted before merger. Being able to rapidly provide an estimate of the sky location is particularly important for multi-messenger astronomy (B. Abbott et al., 2017d). After the detection of GW150914, the estimate of its sky location was shared with 63 ground- and space-based 


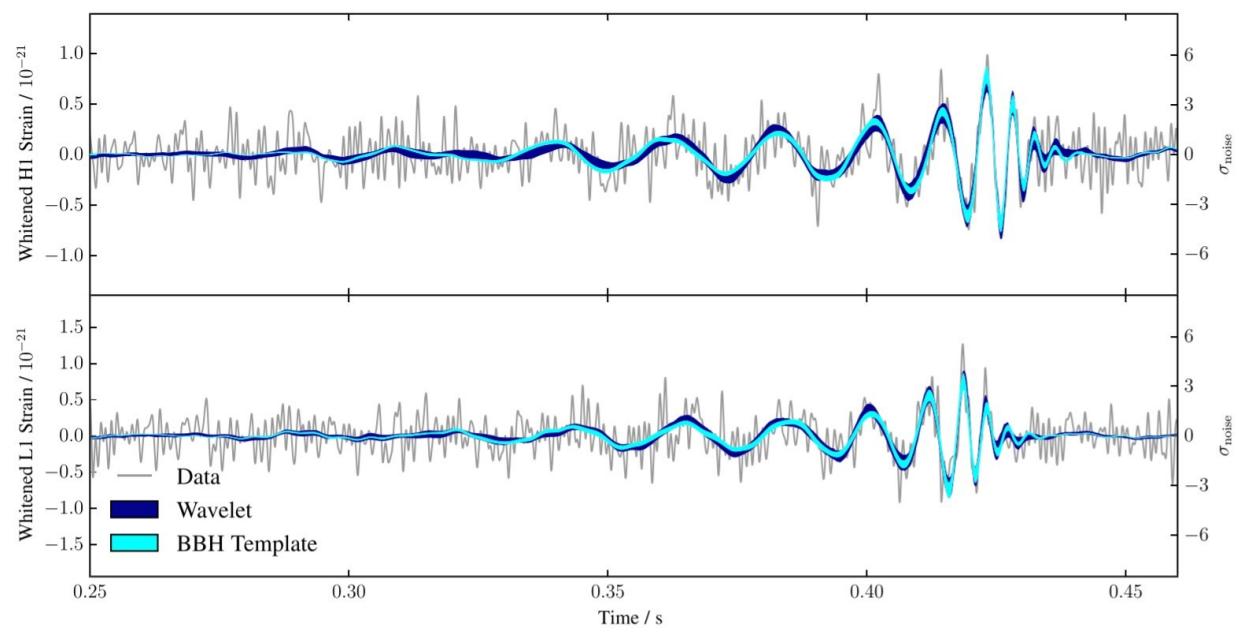

Figure 3: The detector strain measurements of GW150914 (B. Abbott et al., 2016c) observed by the LIGO Hanford (H1, top) and Livingston (L1, bottom) detectors in grey. Times are shown relative to September 14, 2015 at 09:50:45 UTC. The cyan curve is the estimated signal using the IMRPhenom and EOBNR waveform templates, the blue curve a signal reconstruction based on BayesWave. Source: B. Abbott et al. (2016d).

observatories covering radio, optical, near-infrared, X-ray, and gamma-ray wavelengths (B. Abbott et al., 2016b). Fast localization of the gravitational wave source allows a targeted follow-up by electromagnetic telescopes as was the case for GW170817. To this end, Bayestar has been developed. Bayestar conditions on fixed values of the intrinsic parameters and computes the posterior of the extrinsic parameters. This allows an approximation of the marginal posterior distribution of the sky location via numerical integration (L. P. Singer $\&$ Price, 2016). This can provide reliable sky localization and distance estimations within minutes after detection. Bayestar gave an initial estimate of the position in the sky of GW170817 of $31 \mathrm{deg}^{2}$ and an estimate of the luminosity distance of 40土 $8 \mathrm{Mpc}$ (LIGO Scientific Collaboration, Virgo Collaboration, 2017). A more accurate estimate of the sky location, $28 \mathrm{deg}^{2}$, was then provided by LALInference (B. Abbott et al., 2017c). Methods for describing the three-dimensional posterior distribution of sky location and distance have been developed (L. Singer et al., 2016; L. P. Singer et al., 2016).

A different approach to modelling the gravitational waves of compact binary coalescences is implemented in the BayesWave algorithm of N. J. Cornish \& Littenberg (2015). It is not based on a physically meaningful gravitational waveform model but aims to reconstruct the shape of any burst signal using wavelets. The BayesWave reconstruction of GW150914 is displayed in Fig. 3. Ghonge et al. (2020) compare its reconstruction properties to the reconstructions via MCMC and NS in LALInference.

Sampling algorithms such as MCMC and NS give generally accurate parameter estimates 
for compact binary inspirals but can be very slow, e.g. it takes days to obtain results for black hole mergers, weeks for neutron star mergers. Efficient but accurate approximative methods (R. Smith et al., 2016; Canizares et al., 2015; L. P. Singer \& Price, 2016) can yield a significant reduction in computation time. Reduced-order models (ROMs) of gravitational waveforms have reduced the computational cost of Bayesian inference by a more efficient decomposition of the waveform using analytical insight (Pürrer, 2015). R. Smith et al. (2016) construct a ROM that includes the effects of spin precession, inspiral, merger, and ringdown in compact object binaries utilizing the "IMRPhenomPv2" waveform model. A fast reduced-order quadrature allows to approximate posterior distributions at greatly reduced computational costs. A review of waveform acceleration techniques based on reduced order or surrogate models that speed up parameter estimation is provided in Setyawati et al. (2020). Vinciguerra et al. (2017) exploit the chirping behaviour of compact binary inspirals to sample sparsely for portions where the full frequency resolution is not required, Zackay et al. (2018) and N. Cornish (2013) use relative binning and the heterodyning principle, respectively, for fast likelihood evaluation. Rapid parameter inference methods using grid techniques are also being developed (Pankow et al., 2015; Lange et al., 2018).

To speed up parameter estimation, deep learning approaches, particularly variational autoencoders and convolutional neural networks, have recently been explored (George \& Huerta, 2018; Gabbard et al., 2019; Shen et al., 2019; Chua \& Vallisneri, 2020; S. R. Green et al., 2020). Deep learning approaches train neural networks to learn the posterior through stochastic gradient descent to optimize a loss function. The training samples require only sampling from the prior and the likelihood which is fast. It also has the advantage that training can be performed offline and the estimation of parameters from an observed gravitational wave signal becomes almost instantaneous. These methods are still in their infancy and need to be further developed to be able to handle the full parameter space of binary inspirals and longer duration waveforms from multiple detectors. They hold great promise for low-latency parameter estimation and a fast electromagnetic follow-up.

With a whole catalogue of compact black hole mergers from the first, second, and soon third LIGO - Virgo observations runs, it has now become feasible to infer population properties such as their merger rates, mass spectrum, and spin distribution, as for instance in Stevenson et al. (2017); Fishbach et al. (2018); Wysocki et al. (2019); Chase et al. (2020); Callister et al. (2020); B. Abbott et al. (2019c); R. Smith et al. (2020).

\section{Unmodelled Burst Signals}

There are various potential origins of unmodelled short-duration burst signals with no known closed form, such as pulsar glitches, core collapse supernovae, gamma ray burst engines, and unanticipated sources. Amongst these, gravitational waves from core collapse supernovae (CCSNe) are probably the most promising for observation (Gossan et al., 2015). 
To date, gravitational waves from CCSNe have not been directly observed by the network of terrestrial detectors, Advanced LIGO and Advanced Virgo (B. Abbott et al., 2020e). However, a new era in multimessenger astronomy began with the observation of gravitational waves from a binary neutron star inspiral (GW170817) with associated counterparts observed across the electromagnetic spectrum (B. Abbott et al., 2017c). Much like GW170817, CCSNe are an important source of multimessenger astronomy as they will have associated electromagnetic, as well as neutrino counterparts (Hirata et al., 1987; Bionta et al., 1987). The gravitational wave signal from a CCSN will be of order $1 \mathrm{~s}$ or less. LIGO and Virgo regularly search for gravitational wave signals from CCSN (B. Abbott et al., 2019b, 2020d).

Like neutrinos, gravitational waves are emitted from the core of the progenitor and carry information about the dynamics of the core collapse and shock wave revival mechanism that leads to explosion (Kuroda et al., 2017). However, gravitational waveforms from CC$\mathrm{SNe}$ are analytically intractable due to the complex interplay of general relativity, particle physics, and nuclear physics, meaning template-based search methods like those employed in compact binary coalescence pipelines are currently not possible. Alternative parameter estimation routines are needed.

The first attempt to conduct parameter estimation on CCSN gravitational wave signals was by Summerscales et al. (2008), who used the maximum entropy framework to deconvolve noisy data from multiple detectors to extract a CCSN gravitational wave signal. They made inferences on amplitude and phase parameters using cross correlation between a recovered waveform and a set of competing waveforms from the Ott et al. (2004) waveform catalogue, where a match was defined as the model with the maximum cross correlation to the recovered waveform.

Heng (2009) proposed simplifying the problem using principal component analysis (PCA) to reduce a supernova waveform catalogue parameter space to a small number of basis vectors. Röver et al. (2009) extended on this by creating a Metropolis-within-Gibbs sampler to reconstruct rotating core collapse signals using principal component regression (PCR). They attempted to conduct parameter estimation by matching reconstructed signals to catalogue waveforms using a $\chi^{2}$ distance, but this had limited success. Edwards (2017) extended the PCR Bayesian reconstruction of CCSN signals using a birth-death reversible jump MCMC (RJMCMC) approach, allowing the number of principal components to vary, and making use of model averaging to handle the model selection problem. An example of a reconstructed CCSN waveform from the Dimmelmeier et al. (2008) waveform catalogue can be seen in Figure 4.

Abdikamalov et al. (2014) used matched filtering on their newly created waveform catalogue to infer total angular momentum from rotating CCSN signals with errors up to $20 \%$ for rapidly rotating progenitors and $35 \%$ for slowly rotating cores. They also used nested sampling to classify precollapse differential rotation profile, with reasonable success.

Edwards et al. (2014) demonstrated that it is possible to extract astrophysically meaningful 


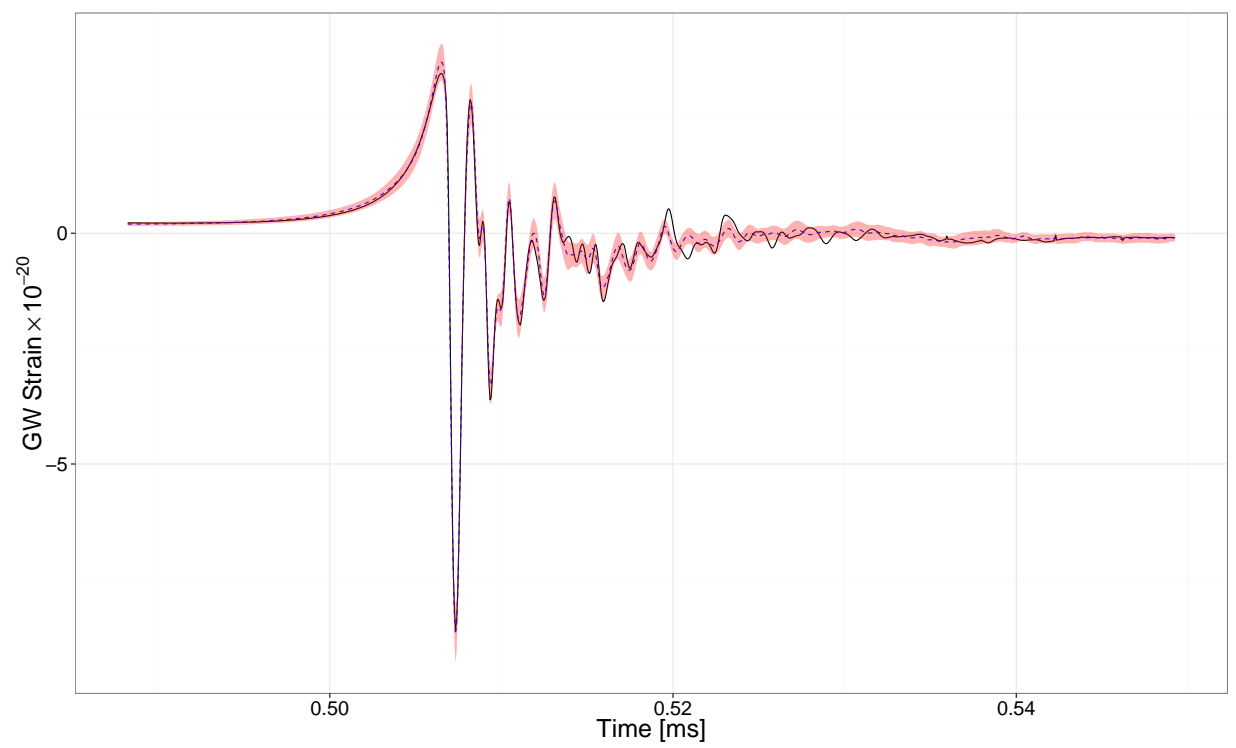

Figure 4: Reconstructed CCSN signal from the Dimmelmeier et al. (2008) waveform catalogue using a transdimensional PCR model. The true signal (solid black) and modelaveraged reconstruction (dashed blue) are overlaid with the model-averaged $90 \%$ credible region (shaded pink).

information encoded in the posterior principal component coefficients in the Bayesian PCR model of Röver et al. (2009). Using posterior predictive sampling, they were able to give Bayesian credible intervals for the first time on parameters such as the ratio of rotational kinetic energy to gravitational potential energy of the inner core at bounce. The authors also used supervised machine learning methods to classify the precollapse differential rotation profile.

Engels et al. (2014) used frequentist multivariate regression and classical hypothesis testing to analyse important astrophysical parameters from CCSNe signals. In contrast to the PCR approach of Heng (2009); Röver et al. (2009) to reconstruct waveforms, the authors used the method of least squares to find an encoded relationship between the PC basis functions and astrophysical parameters, identifying the most important astrophysical parameters from signals buried in simulated detector noise.

A recent extension of the PCA-based approach to parameter estimation of CCSN signals has been given by Roma et al. (2019). It includes features in the gravitational wave signal that are associated with g-modes and the standing accretion shock instability. Rather than computing the principal components of the simulated waveform time series, it performs a PCA on the spectrograms and test the performance using simulated data for planned future detectors such as the Einstein Telescope and Cosmic Explorer. 
The supernova explosion mechanism is not fully understood. The two most popular and well-studied supernova explosion mechanisms are the neutrino-driven explosion for nonrotating and slow-rotating progenitors and magnetorotational-driven explosion for rapidly rotating progenitors (Janka, 2012). As the gravitational wave signals from these explosion mechanisms are morphologically different, this has been an area of much focus for parameter estimation studies. This was first formulated as a classification problem by Logue et al. (2012), where they used PCR and nested sampling (Skilling, 2006), computing Bayesian evidence to select the most likely explosion mechanism. Classifying the supernova explosion mechanism using nested sampling has been further studied by e.g., Powell et al. (2016) using real detector noise, Powell et al. (2017) for 3D simulations and noise transient rejection.

M. Coughlin et al. (2014), B. Abbott et al. (2019b) and Banagiri et al. (2020) developed Bayesian approaches for estimating the parameters of transient signals based on the timefrequency maps. Lynch et al. (2017) explored an information-theoretic approach to the burst detection problem. Both used nested sampling as part of an algorithm for detecting short-duration gravitational wave bursts. The method of M. Coughlin et al. (2014) can also be used for long-duration gravitational wave transients, possibly lasting up to thousands of seconds.

One of the most popular and sophisticated methods for constructing unmodelled bursts is the BayesWave algorithm by N. J. Cornish \& Littenberg (2015). BayesWave uses MorletGabor continuous wavelets to construct bursts and glitches. Under the reversible jump MCMC framework (P. Green, 1995), they treat the number of wavelets as variable. Parameter estimation routines for unmodelled bursts with BayesWave are currently being developed, with reasonable success at sky localisation (Bécsy et al., 2017).

In line with the current trend in gravitational wave data analysis, deep learning methods are being explored. Similar to the deep learning methods for glitch characterisation (Zevin et al., 2017; George et al., 2018), convolutional neural networks have started populating the CCSN parameter estimation literature (see e.g., Astone et al. (2018); Heng \& Messenger (2019); Iess et al. (2020)) due to their success in image classification problems. However, deep learning approaches for CCSNe are still in their infancy and have not developed beyond classification problems.

\section{Continuous Signals}

Various sources, such as binary systems far from coalescence or non-axisymmetric rotating neutron stars will emit continuous gravitational waves. Gravitational wave signals from rapidly rotating biaxial or triaxial neutron stars, so-called pulsars, have been searched for but have not yet been observed by Advanced LIGO - Advanced Virgo (B. Abbott et al., 2017b, 2019e,g,h,a). Pulsars emit gravitational waves that will likely be seen on Earth as 
weak continuous signals and are promising candidates for future observations runs. These quasi-periodic signals are of long duration with near constant amplitude and frequency. The gravitational wave signal from such an object is at twice its rotation frequency $f_{s}=2 f_{r}$. Identification of a periodic gravitational wave signal is challenging because of the weakness of the signal. But radio observations can provide information about the sky location, rotation frequency, and spindown rate of known pulsars, thus allowing a targeted search in a very narrow spectral window (Dupuis \& Woan, 2005). The observed signal, described by

$$
h(t)=F_{+}(t, \psi) h_{0} \frac{1}{2}\left(1+\cos ^{2} \iota\right) \cos \phi(t)+F_{\times}(t, \psi) h_{0} \cos \iota \sin \phi(t),
$$

depends on several unknown parameters: the overall amplitude of the gravitational wave signal $h_{0}$, the polarization angle $\psi$, the angle $\iota$ between spin axis of the pulsar and the line of sight, and the phase evolution $\phi(t)$. The response of the detector to the two polarization is given by $F_{+}(t, \psi)$ and $F_{\times}(t, \psi)$. The sky position parameters $\alpha$ and $\delta$ are fixed. A simple slowdown model provides the rotational phase evolution of the signal via a short Taylor series expansion

$$
\phi(t)=\phi_{0}+2 \pi\left[f_{\mathrm{s}}\left(T-T_{0}\right)+\frac{1}{2} \dot{f}_{\mathrm{s}}\left(T-T_{0}\right)^{2}+\frac{1}{6} \ddot{f}_{\mathrm{s}}\left(T-T_{0}\right)^{3}+\ldots\right]
$$

where $T=t+\delta t=t+\frac{\vec{r} \cdot \vec{n}}{c}+\Delta T$ is the time of arrival of the signal at the solar system barycenter, $\phi_{0}$ is the phase of the signal at a fiducial time $T_{0}, \vec{r}$ is the position of the detector with regard to the solar system barycenter, $\vec{n}$ is a unit vector in the direction of the pulsar, $c$ is the speed of light, and $\Delta T$ contains the relativistic corrections to the arrival time (Christensen, Dupuis, et al., 2004). For most pulsars, the time derivative $\dot{f}_{\mathrm{s}}$ is very small and $\ddot{f}_{\mathrm{s}}$ is often swamped by timing noise. If $f_{\mathrm{s}}$ and $\dot{f}_{\mathrm{s}}$ are known from radio observations, the signal can be heterodyned by multiplying the data by $\exp [-i \phi(t)]$, low-pass filtered and resampled, yielding a simple model with four unknown parameters $h_{0}, \psi, \iota, \phi_{0}$. If there is an uncertainty in the frequency and frequency derivative then we have two additional parameters, the differences between the signal and heterodyne frequency and frequency derivatives (Christensen, Dupuis, et al., 2004; Umstätter et al., 2004; Dupuis \& Woan, 2005). Early MCMC techniques for sampling from the posterior distribution used a combination of reparametrizaton, delayed rejection, and simulated annealing Umstätter et al. (2004). Nested sampling code for parameter estimation and model selection in targeted searches for continuous gravitational wave signals from pulsars has been developed by Pitkin et al. (2012) and implemented in the LALInference software and is described in detail in Pitkin et al. (2017). Ashton \& Prix (2018) introduce a method for the hierarchical follow-up of continuous gravitational wave candidates by leveraging MCMC optimization of the F-statistic using the affine-invariant ensemble sampler (Foreman-Mackey et al., 2013).

Dreissigacker et al. (2019) highlighted that deep learning, particularly convolutional neural networks, can be used to directly search for continuous waves. Though the results were much faster than matched filtering, the approach needs to be further developed to be more competitive (in terms of the detection probability) with existing methods. 


\section{Stochastic Gravitational Wave Background}

The combination of all gravitational wave signals that can not be individually resolved will make up what is called the stochastic gravitational wave background (SGWB) (Romano \& Cornish, 2017; Christensen, 2019). Analogous to the cosmic microwave background, the physical processes in the early evolution of the Universe created the cosmological SGWB. The superposition of many unresolved signals from many independent sources such as the galactic population of white dwarf binaries, compact binary mergers, supernovae, pulsars, magnetars, and cosmic strings make up the astrophysical SGWB. As electromagnetic waves cannot provide information about astrophysical sources and processes any earlier than 400,000 years after the Big Bang - the time of last scattering - detection and estimation of the SGWB is extremely important to probe the early Universe. Unlike transient gravitational wave signals that come from a certain location in the sky, the SGWB signal will come from all directions and may or may not be isotropic and uniformly distributed across the sky (B. Abbott et al., 2017f, 2019). By and large, the SGWB is a stochastic signal and will be another source of noise in a single detector, often modelled as stationary Gaussian with mean zero and positive definite covariance matrix or spectral density to be estimated. So the fundamental problem is to distinguish the SGWB "noise" from instrumental noise (M. Adams \& Cornish, 2010). When there are several detectors such as in the network of Advanced LIGO - Advanced Virgo, cross-correlation methods can be employed, e.g. with observations at two detectors

$$
d^{(k)}(t)=h(t)+n^{(k)}(t), \quad k=1,2
$$

assuming independent noise components, the correlation between the observations becomes (Christensen, 1992):

$$
\operatorname{Cov}\left(d^{(1)}(t), d^{(2)}(t)\right)=\operatorname{Cov}\left(h(t)+n^{(1)}(t), h(t)+n^{(2)}(t)\right)=\operatorname{Cov}(h(t), h(t)) .
$$

Advanced LIGO and Advanced Virgo have used these correlation methods to search for the SGWB. Even though no SGWB signal has been detected to date, upper limits have been placed on the energy density of the SGWB within the frequency range of $20 \mathrm{~Hz}$ to 1000 $\mathrm{Hz}$ (B. Abbott et al., 2017f, 2019i). The energy density of the SGWB is assumed to have the form

$$
\Omega_{G W}(f)=\Omega_{\alpha}\left(\frac{f}{f_{r e f}}\right)^{\alpha}
$$

$\Omega_{G W}(f)$ is the power spectral density of the SGWB divided by the closure density of the universe $\rho_{c}=3 H_{0}^{2} /(8 \pi G)$, and the Hubble constant is $H_{0}=67.9 \mathrm{~km} \mathrm{~s}^{-1} \mathrm{Mpc}^{-1}$ (Ade et al., 2016). The slope of the energy spectral density $\alpha$ is assumed to be zero for a cosmological background, and 2/3 for a background created by binary black hole and binary neutron star mergers throughout the history of the universe. A reference frequency $f_{\text {ref }}$ defines where the amplitude $\Omega_{\alpha}$ is measured and reported. In the latest results reported by LIGO and Virgo based on the observations from their second observing run there was 
no detected SGWB, and parameter estimation methods were used to generate posterior distribution functions for $\Omega_{\alpha}$ and $\alpha$. The Bayesian parameter estimation method used by LIGO-Virgo for the SGWB search was first presented by Mandic et al. (2012). For the constraint of $\alpha=0$, a presumed cosmological background, a 95\% credible level upper limit was set at $25 \mathrm{~Hz}$ to be $\Omega_{0}<6.0 \times 10^{-8}$, while for a compact binary produced background with $\alpha=2 / 3$ the upper limit for $f_{r e f}=25 \mathrm{~Hz}$ is $\Omega_{2 / 3}<4.8 \times 10^{-8}$ (B. Abbott et al., 2019i).

General relativity predicts that the polarization of gravitational waves would only have a tensor form, while alternate theories of gravity predict vector and scalar polarizations. LIGO and Virgo have searched for the presence of these alternate polarizations, and have not found a signal. Using nested sampling, a Bayes factor has been used to compare the presence of a signal to Gaussian noise. Bayes factors have been computed in searches for all three polarizations, and for the results to date for LIGO and Virgo no SGWB of any polarization is observed (Callister et al., 2017; B. Abbott et al., 2018b, 2019i).

The future space-based interferometer space antenna LISA will search for a SGWB in the $10^{-5} \mathrm{~Hz}$ to $10^{-1} \mathrm{~Hz}$ frequency band (Amaro-Seoane et al., 2017). LISA will yield obserations from thousands of sources of which many will remain unresolved such as gravitational waves from the galactic white dwarf binaries which will form a foreground and in addition to the instrument noise will make the estimation of the SGWB even more difficult (Barack \& Cutler, 2004; M. R. Adams \& Cornish, 2014; Sachdev et al., 2020). LISA is comprised of three coupled interferometers. A principal component-like combination of the three output signals is made in order to eliminate the effect of this correlated noise. This is called time delay interferometry (TDI) (Vinet, 2013; Tinto \& Dhurandhar, 2014). When expressed as the orthogonal modes $(A, E, T)$, one of them, $T$, is insensitive to the gravitational wave signal at low frequencies; this channel can be used to understand the noise. The other two channels are orthogonal. This means that auto-correlation methods must be used to try to observe the SGWB (Romano \& Cornish, 2017; T. L. Smith \& Caldwell, 2019), with the null channel employed to disentangle the detector noise from the SGWB signal. New search strategies and parameter estimation methods such as for instance in Caprini et al. (2019) need to be developed that can be applied to non-Gaussian, anisotropic, circularly polarized backgrounds, and backgrounds with polarization components predicted by alternative theories to general relativity (Romano \& Cornish, 2017). This is currently an active area of research and Bayesian parametric and nonparametric methods for spectral density estimation of time series as reviewed in Section 7 will be important.

\section{Interferometer Noise}

As described in Section 2, for the purpose of estimating transient signals, the power spectral density of the noise $n(t)$ is usually estimated 'off-source', i.e. from a separate stretch of data not containing the signal using Welch's method and is then assumed to be fixed and 
known. For short duration signals, it might be appropriate to assume that the noise process is stationary and that the PSD thus does not change over time, however, for longer duration signals, this is not a reasonable assumption. Similarly, the assumption of Gaussianity is questionable considering the large number of transient noise events, known as glitches, e.g. from environmental sources, weather, or equipment faults. Residuals from parameter estimation using certain noise assumptions need to be carefully checked as for instance described in B. Abbott et al. (2020a). Even if the noise is stationary, estimating the PSD from a separate data sequence and then assuming it to be fixed for the purpose of signal parameter estimation ignores any uncertainty in the PSD estimate and can thus lead to biased signal parameter estimates (Chatziioannou et al., 2019).

For an accurate estimation of signal parameters as well as sensitive and confident signal detection (Venumadhav et al., 2019), it has been realized that a realistic modelling of the interferometer noise is extremely important. Such a noise model should be able to handle non-Gaussian and time-varying noise and to be included in an 'on-source' method, i.e. a method that estimates both signal and noise parameters simultaneously. Various approaches have been suggested in the literature that achieve some of these goals.

To be able to track changes in the PSD over a long period of time, Cuoco (2001) suggested to split the time series into smaller chunks and estimate the PSD using classical parametric spectral density estimation methods based on fitting autoregressive (AR) or autoregressive moving average models. Zackay et al. (2019) deal pragmatically with alleviating the effect of non-stationary noise on signal detection by dividing the matched filtering overlaps by their locally estimated standard deviations.

For stationary Gaussian noise $\mathbf{n}$ with an unknown PSD, a prior model for $S(f)$ in the Whittle likelihood (4):

$$
L(\mathbf{n}) \approx \frac{1}{\operatorname{det}(\pi T \mathbf{S})} \mathrm{e}^{-\frac{1}{T} \tilde{n}^{*} \mathbf{S}^{-1} \tilde{n}}=\exp \left\{-\sum_{j}\left[\frac{\tilde{n}\left(f_{j}\right)^{2}}{T S\left(f_{j}\right)}+\log \left(\pi T S\left(f_{j}\right)\right)\right]\right\}
$$

needs to be specified. Röver et al. (2011) modeled the unknown spectral density components using conjugate inverse Gamma distributions, yielding Student-t marginal distributions for the errors and enabling to accommodate outliers in the data. Similarly, T. B. Littenberg et al. (2013) and Veitch et al. (2015) incorporate uncertainty about the estimated PSD by an additional scale factor $\eta_{j}$ for each frequency bin, i.e. replacing $S\left(f_{j}\right)$ by $\eta_{j} S\left(f_{j}\right)$, and giving it a Normal prior with mean 1, where $S\left(f_{j}\right)$ is estimated beforehand using the Welch method. BayesLine, a flexible Bayesian spectral density estimation method for Gaussian stationary noise that has been widely applied for gravitational wave data analysis was developed by T. B. Littenberg \& Cornish (2015). BayesLine models the smooth part of the PSD by a linear combination of cubic splines where the number as well as the knots of the basis splines are unknown parameters. The spectral lines in the PSD are modeled using a sum of Lorentzians where the number, location and line width are unknown parameters. A reversible jump MCMC algorithm is used to sample from the posterior distribution. This 


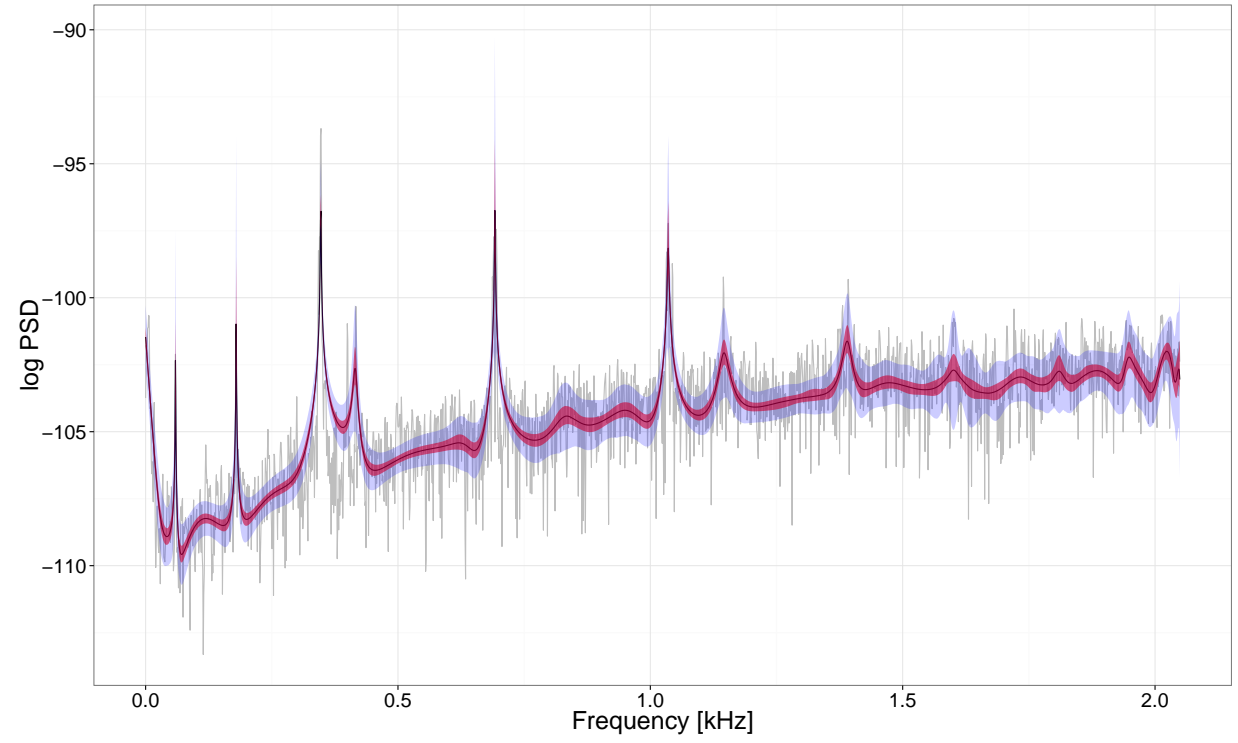

Figure 5: Estimated log spectral density for a $1 \mathrm{~s}$ segment of Advanced LIGO S6 data. The posterior median log spectral density estimate using the corrected likelihood with an AR(35) working model (solid black), pointwise 90\% credible region (shaded red), and uniform $90 \%$ credible region (shaded violet) are overlaid with the log periodogram (grey) (Kirch et al., 2019).

off-source algorithm is then extended in N. J. Cornish \& Littenberg (2015) to an on-source method, known as BayesWave, by simultaneously fitting a gravitational wave burst signal and potential glitches, both modeled as a sum of Morlet-Gabor continuous wavelets, see also Section 4. Whereas BayesWave can reconstruct the gravitational wave signal as demonstrated for instance in Figure 3 it does not provide estimates of the physically meaningful waveform parameters. Biscoveanu et al. (2020) combined the BayesLine model for the PSD with the physical CBC waveform models to simultaneously estimate the signal parameters and the PSD. By marginalizing over the PSD, the marginal posterior distributions of the signal parameters take the full uncertainty of the PSD estimates into account.

Instead of simultaneously estimating the spectral density and waveform parameters Talbot \& Thrane (2020) developed a variant of Welch method by taking the median instead of the average of periodograms over neighbouring segments. The likelihood is derived after marginalization over the uncertainty in the median PSD estimate. The analysis is shown to be robust with respect to large outliers.

Approaches based on a parametric model for the spectral density, such as those based on fitting autoregressive moving average (ARMA) models, can be very efficient when the parametric model is correctly specified but can lead to biased results under model misspecification. Nonparametric models, on the other hand, have much wider applicability 
and robustness as they do not rely on finite-dimensional distributional assumptions. A nonparametric on-source approach, treating the spectral density function $S(f)$ as an infinitedimensional parameter and modelling this nonparametrically using a Bernstein-Dirichlet process prior (Choudhuri et al., 2004) has been developed by Edwards et al. (2015) and used to simultaneously fit rotating core collapse supernova gravitational wave burst signals embedded in simulated Advanced LIGO - Advanced Virgo noise. To improve the approximation of spectral lines, this nonparametric method was modified to use B-splines instead of Bernstein polynomials. By putting a Dirichlet process prior on the knot differences, the B-spline-Dirichlet process prior was shown to be able to accurately pick up sharp peaks and spectral lines in the data from the LIGO S6 science run (Edwards et al., 2019). The method is implemented in the R package bsplinePsd (Edwards et al., 2018). It has also been used as on on-source model for simultaneously estimating parameters of a non-chirping galactic white dwarf binary signal in simulated LISA data (Edwards et al., 2020). An MCMC algorithm combined with parallel tempering was used for posterior computation. A recent modification that reduces the computational complexity while keeping the good approximation and coverage properties of the B-splines by using P-splines, i.e. B-splines but with fixed knots and a smoothness penalty on the coefficients, is given in Maturana-Russel \& Meyer (2019) and implemented in the R package psplinePsd (Maturana-Russel \& Meyer, 2020). By taking advantage of a well-fitting parametric autoregressive model, Kirch et al. (2019) can improve on the Whittle likelihood approximation using a nonparametric correction of a parametric working model and prove posterior consistency. Using the Bernstein-Dirichlet process prior for the spectral density, they demonstrate improved performance using the same S6 LIGO noise data as Edwards et al. (2019). A spectral density estimate based on the corrected likelihood and an autoregressive working model is shown in Figure 5. Sampling is based on adaptive Metropolis-Hastings steps within the Gibbs sampler, implemented in the R package beyondWhittle (Meier et al., 2018). These nonparametric approaches to spectral density estimation can be used to simultaneously estimate the waveform parameters in a Gibbs step, as demonstrated for instance in Edwards et al. (2015).

\section{Conclusion}

Bayesian methods for parameter estimation of gravitational wave data have proven to be essential and effective for analysing the events detected by Advanced LIGO and Advanced Virgo (B. Abbott et al., 2019d), and will play an equally important role for the future spacebased detector LISA (Amaro-Seoane et al., 2017). The two mainstays of posterior computational techniques that are routinely used and implemented in LALInference (Veitch et al., 2015) are parallel tempering MCMC and nested sampling. The main problems with either algorithm when exploring the high-dimensional parameter space are potentially getting stuck in local maxima and slow mixing. These are due to the multimodality of the posterior distribution and the inherent sequential Markov chain steps of both algorithms that yield 
slow convergence when there are high correlations between the parameters. Any convergence acceleration methods that can yield better mixing, e.g. via adaptive MCMC methods (Barber et al., 2011) and enhancements of nested sampling (Feroz \& Skilling, 2013; Brewer $\&$ Foreman-Mackey, 2018) will be critical. Reduced order models and surrogate waveform models (R. Smith et al., 2016; Setyawati et al., 2020) have and will continue to play a role in the acceleration of posterior computations. Furthermore, the development of nonparametric approaches will allow to make the inference more robust with respect to certain assumptions.

An alternative inferential framework which holds great promise for the future of gravitational wave analysis and has seen an enormous increase in research activity is deep learning. This is a machine learning technique that is extremely scalable and can learn from raw data by using deep hierarchical layers of neural networks combined with optimization techniques based on back-propagation and gradient descent (Goodfellow, 2016). It also allows to take account of deviations from the usual assumption of stationary Gaussian noise, as it can be trained on signals embedded in non-Gaussian and non-stationary noise. Whereas deep learning has so far been mainly employed for detection and classification problems, see e.g. Gabbard et al. (2018); George et al. (2018); Gebhard et al. (2019); S. Coughlin et al. (2019); Corizzo et al. (2020); Beheshtipour \& Papa (2020); Cuoco et al. (2020), recent research has a focus on the parameter estimation problem (George \& Huerta, 2018; Fan et al., 2019; Chua \& Vallisneri, 2020). Its main advantage is the fact that the timeconsuming training of the neural nets can be performed off-line and then potentially render the parameter estimates of an observed gravitational event in an instant.

In their third observing run, O3, Advanced LIGO and Advanced Virgo reported potential detections at a cadence of about one per week (LIGO Scientific Collaboration, Virgo Collaboration, 2020a). This rate will increase in the upcoming observing runs as the sensitivity of the detectors improves (B. Abbott et al., 2018a). Bayesian parameter estimation will continue play a critically important role in the description of the physical systems that are producing the gravitational wave events.

\section{Funding Information}

RM gratefully acknowledges support by the James Cook Fellowship from Government funding, administered by the Royal Society Te Apārangi and DFG Grant KI 1443/3-2. The work of NC was supported by US NSF grant PHY-1806990.

\section{Acknowledgments}

We thank Claudia Kirch for fruitful discussions regarding nonparametric approaches to

power spectral density estimation. We thank Sharan Banagiri, Christopher Berry, Tito Dal 
Canton, Michael Pürrer, and John Veitch for comments on the manuscript. This paper has been assigned LIGO document P2000177.

\section{References}

Aasi, J., et al. (2013). Parameter estimation for compact binary coalescence signals with the first generation gravitational-wave detector network. Phys. Rev. D, 88, 062001. doi: 10.1103/PhysRevD.88.062001

Aasi, J., et al. (2015). Advanced LIGO. Class. Quant. Grav., 32, 074001. doi: 10.1088/ 0264-9381/32/7/074001

Abbott, B., Abbott, R., Abbott, T. D., Abernathy, M. R., Acernese, F., Ackley, K., ... et al. (2016, Jun). Characterization of transient noise in advanced ligo relevant to gravitational wave signal gw150914. Classical and Quantum Gravity, 33(13), 134001. Retrieved from http://dx.doi.org/10.1088/0264-9381/33/13/134001 doi: 10.1088/ 0264-9381/33/13/134001

Abbott, B., Bloemen, S., Groot, P., Nelemans, G., \& Schmidt, P. (2019). Search for the isotropic stochastic background using data from advanced ligo's second observing run. Physical Review D, 100, 1-16.

Abbott, B., et al. (2016a). GW150914: First results from the search for binary black hole coalescence with Advanced LIGO. Phys. Rev. D, 93(12), 122003. doi: 10.1103/ PhysRevD.93.122003

Abbott, B., et al. (2016b). Localization and broadband follow-up of the gravitationalwave transient gw150914. Retrieved from http://eprints.whiterose.ac .uk/107663/1/1602.08492v4.pdf

Abbott, B., et al. (2016c). Observation of Gravitational Waves from a Binary Black Hole Merger. Phys. Rev. Lett., 116(6), 061102. doi: 10.1103/PhysRevLett.116.061102

Abbott, B., et al. (2016d). Properties of the Binary Black Hole Merger GW150914. Phys. Rev. Lett., 116(24), 241102. doi: 10.1103/PhysRevLett.116.241102

Abbott, B., et al. (2017a). Exploring the sensitivity of next generation gravitational wave detectors. Classical and Quantum Gravity, 34(4), 044001.

Abbott, B., et al. (2017b). First search for gravitational waves from known pulsars with Advanced LIGO. The Astrophysical Journal, 839(1), 12.

Abbott, B., et al. (2017c). GW170817: Observation of Gravitational Waves from a Binary Neutron Star Inspiral. Phys. Rev. Lett., 119(16), 161101. doi: 10.1103/PhysRevLett.119 .161101 
Abbott, B., et al. (2017d). Multi-messenger observations of a binary neutron star merger. ASTROPHYSICAL JOURNAL LETTERS, 848(2). Retrieved from https://biblio . ugent.be/publication/8620611

Abbott, B., et al. (2017e, October). Multi-messenger Observations of a Binary Neutron Star Merger. Astrophys. J. Lett., 848, L12. doi: 10.3847/2041-8213/aa91c9

Abbott, B., et al. (2017f). Upper Limits on the Stochastic Gravitational-Wave Background from Advanced LIGO's First Observing Run. Phys. Rev. Lett., 118(12), 121101. ([Erratum: Phys. Rev. Lett.119,no.2,029901(2017)]) doi: 10.1103/PhysRevLett.118.121101 ,10.1103/PhysRevLett.119.029901

Abbott, B., et al. (2018a). Prospects for Observing and Localizing Gravitational-Wave Transients with Advanced LIGO, Advanced Virgo and KAGRA. Living Rev. Rel., 21(1), 3. doi: 10.1007/s41114-018-0012-9,10.1007//rr-2016-1

Abbott, B., et al. (2018b). Search for Tensor, Vector, and Scalar Polarizations in the Stochastic Gravitational-Wave Background. Phys. Rev. Lett., 120(20), 201102. doi: 10.1103/PhysRevLett.120.201102

Abbott, B., et al. (2019a). All-sky search for continuous gravitational waves from isolated neutron stars using Advanced LIGO O2 data. Phys. Rev. D, 100(2), 024004. doi: 10 .1103/PhysRevD.100.024004

Abbott, B., et al. (2019b). All-sky search for short gravitational-wave bursts in the second advanced ligo and advanced virgo run. Physical Review D, 100, 1-18.

Abbott, B., et al. (2019c). Binary Black Hole Population Properties Inferred from the First and Second Observing Runs of Advanced LIGO and Advanced Virgo. Astrophys. J. Lett., 882(2), L24. doi: 10.3847/2041-8213/ab3800

Abbott, B., et al. (2019d). GWTC-1: A Gravitational-Wave Transient Catalog of Compact Binary Mergers Observed by LIGO and Virgo during the First and Second Observing Runs. Phys. Rev., X9(3), 031040. doi: 10.1103/PhysRevX.9.031040

Abbott, B., et al. (2019e). Narrow-band search for gravitational waves from known pulsars using the second ligo observing run. Physical Review D, 99, 1-20.

Abbott, B., et al. (2019f). Properties of the binary neutron star merger gw170817. arXiv.org, 9(1). Retrieved from http://search.proquest.com/docview/ $2073341664 /$

Abbott, B., et al. (2019g). Searches for gravitational waves from known pulsars at two harmonics in 2015-2017 ligo data. Retrieved from http://eprints. whiterose .ac.uk/158305/1/Abbott_2019_ApJ_879_10.pdf

Abbott, B., et al. (2019h, Dec). Search for gravitational waves from Scorpius X-1 in the second Advanced LIGO observing run with an improved hidden Markov model. Phys. 
Rev. D, 100, 122002. Retrieved from https://link.aps.org/doi/10.1103/ PhysRevD.100.122002 doi: 10.1103/PhysRevD.100.122002

Abbott, B., et al. (2019i). Search for the isotropic stochastic background using data from Advanced LIGO's second observing run. Phys. Rev., D100(6), 061101. doi: 10.1103/ PhysRevD.100.061101

Abbott, B., et al. (2020a). A guide to LIGO-Virgo detector noise and extraction of transient gravitational-wave signals. Class. Quant. Grav., 37(5), 055002. doi: 10.1088/1361-6382/ ab685e

Abbott, B., et al. (2020b, 4). GW190412: Observation of a Binary-Black-Hole Coalescence with Asymmetric Masses. (accepted PRD)

Abbott, B., et al. (2020c). GW190425: Observation of a Compact Binary Coalescence with Total Mass $\sim 3.4 M_{\odot}$. Astrophys. J. Lett., 892, L3. doi: 10.3847/2041-8213/ab75f5

Abbott, B., et al. (2020d). Optically targeted search for gravitational waves emitted by corecollapse supernovae during the first and second observing runs of advanced LIGO and advanced Virgo. Phys. Rev. D, 101(8), 084002. doi: 10.1103/PhysRevD.101.084002

Abbott, B., et al. (2020e, Apr). Optically targeted search for gravitational waves emitted by core-collapse supernovae during the first and second observing runs of advanced ligo and advanced virgo. Phys. Rev. D, 101, 084002. Retrieved from https:// I ink .aps.org/doi/10.1103/PhysRevD.101.084002 doi: 10.1103/PhysRevD .101 .084002

Abbott, R., Abbott, T., Abraham, S., Acernese, F., Ackley, K., Adams, C., ... ... (2019). Open data from the first and second observing runs of advanced ligo and advanced virgo. arXiv.org. Retrieved from http://search.proquest.com/docview/ $2331358360 /$

Abbott, R., Abbott, T., Abraham, S., Acernese, F., Ackley, K., Adams, C., ... ... (2020). Gw190814: Gravitational waves from the coalescence of a $23 \mathrm{~m}_{\odot}$ black hole with a 2.6 $\mathrm{m}_{\odot}$ compact object. arXiv.org, 896(2). Retrieved from http: // search. proquest .com/docview/2416756076/

Abdikamalov, E., Gossan, S., DeMaio, A. M., \& Ott, C. D. (2014). Measuring the angular momentum distribution in core-collapse supernova progenitors with gravitational waves. Physical Review D, 90, 044001.

Acernese, F., Adams, T., Agatsuma, K., Aiello, L., Allocca, A., Aloy, M. A., ... ... (2018). Calibration of advanced virgo and reconstruction of the gravitational wave signal $\mathrm{h}(\mathrm{t})$ during the observing run o2. Classical and Quantum Gravity, 35(20), 205004.

Acernese, F., et al. (2015). Advanced Virgo: a second-generation interferometric gravita- 
tional wave detector. Class. Quant. Grav., 32(2), 024001. doi: 10.1088/0264-9381/32/2/ 024001

Adams, M., \& Cornish, N. (2010). Discriminating between a stochastic gravitational wave background and instrument noise. arXiv.org, 82(2). Retrieved from http://search .proquest.com/docview/2084838903/

Adams, M. R., \& Cornish, N. J. (2014, Jan). Detecting a stochastic gravitational wave background in the presence of a galactic foreground and instrument noise. Phys. Rev. D, 89, 022001. Retrieved from https://link.aps.org/doi/10.1103/ PhysRevD.89.022001 doi: 10.1103/PhysRevD.89.022001

Ade, P. A. R., et al. (2016). Planck 2015 results. XIII. Cosmological parameters. Astron. Astrophys., 594, A13. doi: 10.1051/0004-6361/201525830

Ali, A., Christensen, N., Meyer, R., \& Rover, C. (2012). Bayesian inference on EMRI signals using low frequency approximations. Class. Quant. Grav., 29, 145014. doi: $10.1088 / 0264-9381 / 29 / 14 / 145014$

Amaro-Seoane, P., et al. (2017, February). Laser Interferometer Space Antenna. ArXiv e-prints.

Anderson, W. G., Brady, P. R., Creighton, J. D. E., \& Flanagan, E. E. (2001, Jan). Excess power statistic for detection of burst sources of gravitational radiation. Phys. Rev. D, 63, 042003. Retrieved from https://link.aps.org/doi/10.1103/ PhysRevD.63.042003 doi: 10.1103/PhysRevD.63.042003

Arnaud, K., et al. (2007). Report on the first round of the Mock LISA data challenges. Class. Quant. Grav., 24, S529-S540. doi: 10.1088/0264-9381/24/19/S16

Ashton, G., et al. (2019). BILBY: A user-friendly Bayesian inference library for gravitational-wave astronomy. Astrophys. J. Suppl., 241(2), 27. doi: 10.3847/1538-4365/ ab06fc

Ashton, G., \& Prix, R. (2018). Hierarchical multi-stage mcmc follow-up of continuous gravitational wave candidates. arXiv.org, 97(10). Retrieved from http://search .proquest.com/docview/2073807679/

Astone, P., Cerdá-Durán, P., Di Palma, I., Drago, M., Muciaccia, F., Palomba, C., \& Ricci, F. (2018, Dec). New method to observe gravitational waves emitted by core collapse supernovae. Phys. Rev. D, 98, 122002. Retrieved from https://Iink.aps.org/ doi/10.1103/PhysRevD.98.122002 doi: 10.1103/PhysRevD.98.122002

Babak, S. (2017, May). "Enchilada" is back on the menu. In Journal of physics conference series (Vol. 840, p. 012026). doi: 10.1088/1742-6596/840/1/012026

Babak, S., et al. (2008). Report on the second Mock LISA Data Challenge. Class. Quant. Grav., 25, 114037. doi: 10.1088/0264-9381/25/11/114037 
Baghi, Q., Thorpe, I., Slutsky, J., Baker, J., Dal Canton, T., Korsakova, N., \& Karnesis, N. (2019). Gravitational-wave parameter estimation with gaps in LISA: a Bayesian data augmentation method. Phys. Rev. D, 100(2), 022003. doi: 10.1103/PhysRevD.100 .022003

Banagiri, S., Coughlin, M. W., Clark, J., Lasky, P. D., Bizouard, M., Talbot, C., .. Mandic, V. (2020). Constraining the Gravitational-Wave Afterglow From a Binary Neutron Star Coalescence. Mon. Not. Roy. Astron. Soc., 492(4), 4945-4951. doi: 10.1093/mnras/ staa181

Barack, L., \& Cutler, C. (2004). Confusion noise from lisa capture sources. Physical Review $D, 70(12)$.

Barber, D., Cemgil, A. T., Chiappa, S., Atchadé, Y., Fort, G., Moulines, E., \& Priouret, P. (2011). Adaptive markov chain monte carlo: theory and methods (Vol. 9780521196765). Cambridge, UK ;: Cambridge University Press.

Bécsy, B., et al. (2017). Parameter Estimation for Gravitational-wave Bursts with the BayesWave Pipeline. The Astrophysical Journal, 839(15).

Beheshtipour, B., \& Papa, M. (2020). Deep learning for clustering of continuous gravitational wave candidates. Physical Review D, 101(6). Retrieved from https://www. scopus.com/inward/record.uri?eid=2-s2.0 $-85083568512 \&$ doi=10.11032 fPhysRevD. $101.064009 \&$ partnerID = $40 \& \mathrm{md} 5=195 \mathrm{~d} 6 \mathrm{ad} 2 \mathrm{e} 68225969 \mathrm{~b} 4542 \mathrm{ba} 843 \mathrm{e} 34 \mathrm{e}$ (cited By 0) doi: 10.1103/PhysRevD.101.064009

Berry, C. P. L., Mandel, I., Middleton, H., Singer, L. P., Urban, A. L., Vecchio, A., ... Veitch, J. (2015). Parameter estimation for binary neutron-star coalescences with realistic noise during the advanced ligo era. The Astrophysical Journal, 804(2), 114.

Bionta, R. M., Blewitt, G., Bratton, C. B., Casper, D., Ciocio, A., Claus, R., ... Wuest, C. (1987, Apr). Observation of a neutrino burst in coincidence with supernova 1987a in the large magellanic cloud. Phys. Rev. Lett., 58, 1494-1496. Retrieved from https://link.aps.org/doi/10.1103/PhysRevLett.58.1494 doi: 10 $.1103 /$ PhysRevLett.58.1494

Biscoveanu, S., Vitale, S., \& Davies, J. (2020). Quantifying the effect of power spectral density uncertainty on gravitational-wave parameter estimation for compact binary sources. arXiv.org. Retrieved from http: // search.proquest.com/docview/ $2389079631 /$

Biwer, C. M., Capano, C. D., De, S., Cabero, M., Brown, D. A., Nitz, A. H., \& Raymond, V. (2019). Pycbc inference: A python-based parameter estimation toolkit for compact binary coalescence signals. Publications of the Astronomical Society of the Pacific, 131(996). 
Bizouard, M.-A., \& Papa, M. A. (2013). Searching for gravitational waves with the LIGO and Virgo interferometers. Comptes Rendus Physique, 14, 352-365. doi: 10.1016/j.crhy .2013 .03 .001

Blanchet, L. (2014). Gravitational radiation from post-newtonian sources and inspiralling compact binaries. Living Reviews in Relativity, 17(1), 1-187.

Brewer, B. J., \& Foreman-Mackey, D. (2018). : Diffusive nested sampling in c++ and python. Journal of Statistical Software, 86(7).

Brügmann, B. (2018). Fundamentals of numerical relativity for gravitational wave sources. Science, 361(6400), 366-371. Retrieved from https://science.sciencemag . org/content/361/6400/366 doi: 10.1126/science.aat3363

Buonanno, A., \& Damour, T. (1999). Effective one-body approach to general relativistic two-body dynamics. Physical Review D, 59(8).

Buonanno, A., Iyer, B. R., Ochsner, E., Pan, Y., \& Sathyaprakash, B. S. (2009, Oct). Comparison of post-newtonian templates for compact binary inspiral signals in gravitational-wave detectors. Phys. Rev. D, 80, 084043. Retrieved from https://link.aps.org/doi/10.1103/PhysRevD.80.084043 doi: 10 $.1103 /$ PhysRevD.80.084043

Cahillane, C., Betzwieser, J., Brown, D., Goetz, E., Hall, E., Izumi, K., ... Weinstein, A. (2017). Calibration uncertainty for advanced ligo's first and second observing runs. arXiv.org, 96(10). Retrieved from http://search.proquest.com/docview/ $2076569375 /$

Callister, T., Biscoveanu, A. S., Christensen, N., Isi, M., Matas, A., Minazzoli, O., ... Thrane, E. (2017). Polarization-based Tests of Gravity with the Stochastic GravitationalWave Background. Phys. Rev., X7(4), 041058. doi: 10.1103/PhysRevX.7.041058

Callister, T., Fishbach, M., Holz, D., \& Farr, W. (2020). Shouts and murmurs: Combining individual gravitational-wave sources with the stochastic background to measure the history of binary black hole mergers. arXiv.org, 896(2). Retrieved from http://search.proquest.com/docview/2384334826/

Canizares, P., Field, S. E., Gair, J., Raymond, V., Smith, R., \& Tiglio, M. (2015). Accelerated gravitational wave parameter estimation with reduced order modeling. Physical review letters, 114(7).

Caprini, C., Figueroa, D., Flauger, R., Nardini, G., Peloso, M., Pieroni, M., .. . Tasinato, G. (2019). Reconstructing the spectral shape of a stochastic gravitational wave background with lisa. arXiv.org, 2019(11). Retrieved from http://search.proquest.com/ docview/2245972675/

Chase, K., Talbot, C., Berry, C., Carney, M., Zevin, M., Thrane, E., \& Kalogera, V. (2020). Black hole genealogy: Identifying hierarchical mergers with gravitational 
waves. arXiv.org. Retrieved from http://search.proquest.com/docview/ $2397928299 /$

Chatziioannou, K., Haster, C.-J., Littenberg, T. B., Farr, W. M., Ghonge, S., Millhouse, M., ... Cornish, N. (2019). Noise spectral estimation methods and their impact on gravitational wave measurement of compact binary mergers. Physical Review D, 100(10).

Choudhuri, N., Ghosal, S., \& Roy, A. (2004). Bayesian estimation of the spectral density of a time series. Journal of the American Statistical Association, 99(468), 1050 1059. Retrieved from http://www.tandfonline.com/doi/abs/10.1198/ 016214504000000557

Christensen, N. (1992, Dec). Measuring the stochastic gravitational-radiation background with laser-interferometric antennas. Phys. Rev. D, 46, 5250-5266. Retrieved from https://link.aps.org/doi/10.1103/PhysRevD.46.5250 doi: 10.1103/ PhysRevD.46.5250

Christensen, N. (2019). Stochastic gravitational wave backgrounds. Reports on Progress in Physics, 82(1), 016903.

Christensen, N., Dupuis, R., Woan, G., \& Meyer, R. (2004). Metropolis-hastings algorithm for extracting periodic gravitational wave signals from laser interferometric detector data. Physical review., 70(2), 022001-1-022001-7.

Christensen, N., \& Meyer, R. (1998, Sep). Markov chain monte carlo methods for bayesian gravitational radiation data analysis. Phys. Rev. D, 58, 082001. Retrieved from https://link.aps.org/doi/10.1103/PhysRevD.58.082001 doi: 10.1103/PhysRevD.58.082001

Christensen, N., \& Meyer, R. (2001, May). Using markov chain monte carlo methods for estimating parameters with gravitational radiation data. Phys. Rev. D, 64, 022001. Retrieved from https://link.aps.org/doi/10.1103/PhysRevD .64 .022001 doi: 10.1103/PhysRevD.64.022001

Christensen, N., Meyer, R., \& Libson, A. (2004). A metropolis-hastings routine for estimating parameters from compact binary inspiral events with laser interferometric gravitational radiation data. Classical and Quantum Gravity, 21(1), 317-330. doi: 10.1088/0264-9381/21/1/023

Chua, A., \& Vallisneri, M. (2020). Learning bayesian posteriors with neural networks for gravitational-wave inference. arXiv.org, 124(4). Retrieved from http://search .proquest.com/docview/2291096238/

Corizzo, R., Ceci, M., Zdravevski, E., \& Japkowicz, N. (2020). Scalable auto-encoders for gravitational waves detection from time series data. Expert Systems with Applications, 151. Retrieved from https://www.scopus.com/inward/record .uri?eid=2-s2.0-85082875770\&doi=10.1016\%2fj.eswa.2020 
. 113378 \&partner ID $=40$ \&md5 =ce $1301923 \mathrm{ac} 8 \mathrm{db} 7$ f 28 e $880 \mathrm{~b} 801 \mathrm{~b} 1 \mathrm{bc} 28$

(cited By 0) doi: 10.1016/j.eswa.2020.113378

Cornish, N. (2013). Fast fisher matrices and lazy likelihoods. arXiv.org. Retrieved from http://search.proquest.com/docview/2085650762/

Cornish, N., \& Robson, T. (2017, may). Galactic binary science with the new LISA design. Journal of Physics: Conference Series, 840, 012024. Retrieved from https://doi.org/10.1088\%2F1742-6596\%2F840\%2F12F012024 doi: $10.1088 / 1742-6596 / 840 / 1 / 012024$

Cornish, N., \& Shuman, K. (2020). Black hole hunting with lisa. arXiv.org, 101(12). Retrieved from http://search.proquest.com/docview/2400019695/

Cornish, N. J., \& Crowder, J. (2005). LISA data analysis using MCMC methods. Phys. Rev. D, 72, 043005. doi: 10.1103/PhysRevD.72.043005

Cornish, N. J., \& Littenberg, T. B. (2015). BayesWave: Bayesian Inference for Gravitational Wave Bursts and Instrument Glitches. Class. Quant. Grav., 32(13), 135012. doi: $10.1088 / 0264-9381 / 32 / 13 / 135012$

Coughlin, M., Christensen, N., Gair, J., Kandhasamy, S., \& Thrane, E. (2014). Method for estimation of gravitational-wave transient model parameters in frequency-time maps. Classical and Quantum Gravity, 31(16), 165012.

Coughlin, S., Bahaadini, S., Rohani, N., Zevin, M., Patane, O., Harandi, M., ... Kalogera, V. (2019). Classifying the unknown: Discovering novel gravitational-wave detector glitches using similarity learning. Physical Review D, 99(8). Retrieved from https://www. scopus.com/inward/record.uri?eid=2-s2.0 $-85065092821 \& d o i=10.1103 \% 2$ fPhysRevD $.99 .082002 \&$ partnerID $=$ $40 \& \mathrm{md} 5=\mathrm{a} 1477 \mathrm{f} 285 \mathrm{c} 26 \mathrm{~d} 7 \mathrm{abe} 4727 \mathrm{ae} 88 \mathrm{f357f0d}$ (cited By 2) doi: 10.1103/PhysRevD.99.082002

Covas, P. B., Effler, A., Goetz, E., Meyers, P. M., Neunzert, A., Oliver, M., ... Zweizig, J. (2018, Apr). Identification and mitigation of narrow spectral artifacts that degrade searches for persistent gravitational waves in the first two observing runs of advanced ligo. Phys. Rev. D, 97, 082002. Retrieved from https://link.aps.org/doi/ 10.1103/PhysRevD.97.082002 doi: 10.1103/PhysRevD.97.082002

Crowder, J., \& Cornish, N. (2007). A Solution to the Galactic Foreground Problem for LISA. Phys. Rev. D, 75, 043008. doi: 10.1103/PhysRevD.75.043008

Cuoco, E. (2001). On-line power spectra identification and whitening for the noise in interferometric gravitational wave detectors. Classical and Quantum Gravity, 18(9), 17271751.

Cuoco, E., Powell, J., Cavaglià, M., Ackley, K., Bejger, M., Chatterjee, C., ... Williams, 
D. (2020). Enhancing gravitational-wave science with machine learning. arXiv.org. Retrieved from http://search.proquest.com/docview/2401519545/

Cutler, \& Flanagan. (1994). Gravitational waves from merging compact binaries: How accurately can one extract the binary's parameters from the inspiral waveform? Physical review. D, Particles and fields, 49(6), 2658-2697. Retrieved from http://search .proquest.com/docview/1859262384/

Dahal, P. K. (2020). Review of pulsar timing array for gravitational wave research. Journal of astrophysics and astronomy., 41(1).

Damour, T. (2016, Nov). Gravitational scattering, post-minkowskian approximation, and effective-one-body theory. Phys. Rev. D, 94, 104015. Retrieved from https: / / i ink .aps.org/doi/10.1103/PhysRevD.94.104015 doi: 10.1103/PhysRevD.94 .104015

Dimmelmeier, H., Ott, C. D., Marek, A., \& Janka, H.-T. (2008). The gravitational wave burst signal from core collapse of rotating stars. Physical Review D, 78, 064056.

Dreissigacker, C., Sharma, R., Messenger, C., Zhao, R., \& Prix, R. (2019, Aug). Deeplearning continuous gravitational waves. Phys. Rev. D, 100, 044009. Retrieved from https://link.aps.org/doi/10.1103/PhysRevD.100.044009 doi: 10 .1103/PhysRevD.100.044009

Dupuis, R. J., \& Woan, G. (2005, Nov). Bayesian estimation of pulsar parameters from gravitational wave data. Physical Review D, 72(10). Retrieved from http: / / dx . doi .org/10.1103/PhysRevD.72.102002 doi: 10.1103/physrevd.72.102002

Edwards, M. C. (2017). Bayesian modelling of stellar core collapse gravitational wave signals and detector noise (Unpublished doctoral dissertation). Department of Statistics, University of Auckland.

Edwards, M. C., Maturana-Russel, P., Meyer, R., Gair, J., Korsakova, N., \& Christensen, N. (2020). Identifying and addressing nonstationary lisa noise.

Edwards, M. C., Meyer, R., \& Christensen, N. (2014). Bayesian parameter estimation of core collapse supernovae using gravitational wave simulations. Inverse Problems, 30(11). doi: 10.1088/0266-5611/30/11/114008

Edwards, M. C., Meyer, R., \& Christensen, N. (2015). Bayesian semiparametric power spectral density estimation with applications in gravitational wave data analysis. arXiv.org, 92(6). Retrieved from http://search.proquest.com/docview/ $2083264447 /$

Edwards, M. C., Meyer, R., \& Christensen, N. (2018). bsplinepsd: Bayesian nonparametric spectral density estimation using b-spline priors [Computer software manual]. Retrieved from https://CRAN.R-project.org/package=bsplinePsd (R package version 0.6.0) 
Edwards, M. C., Meyer, R., \& Christensen, N. (2019). Bayesian nonparametric spectral density estimation using b-spline priors. Statistics and Computing, 29(1), 67-78.

Einstein, A. (1916). "Die Grundlage der allgemeinen Relativitätstheorie. Annalen der Physik, 49, 769.

Eisenstein, R. A. (2019). Numerical relativity and the discovery of gravitational waves. Annalen der Physik, 531(8), 1800348. Retrieved from https:// onlinelibrary.wiley.com/doi/abs/10.1002/andp.201800348 doi: 10.1002/andp.201800348

Engels, W. J., Frey, R., \& Ott, C. D. (2014). Multivariate Regression Analysis of Gravitational Waves from Rotating Core Collapse. Phys. Rev. D, 90(12), 124026. doi: 10.1103/PhysRevD.90.124026

Fan, X., Li, J., Li, X., Zhong, Y., \& Cao, J. (2019). Applying deep neural networks to the detection and space parameter estimation of compact binary coalescence with a network of gravitational wave detectors. Science China: Physics, Mechanics and Astronomy, 62(6). Retrieved from https://www.scopus.com/inward/ record.uri?eid=2-s2.0-85061975292\&doi=10.1007\%2fs11433-018 $-9321-7$ \&partner $\mathrm{ID}=40$ \&md5=cbe 0 ee $12526776 \mathrm{bc} 4 \mathrm{~b} 18056911 \mathrm{fd} 2 \mathrm{a} 20$ (cited By 6) doi: 10.1007/s11433-018-9321-7

Farr, B., Kalogera, V., \& Luijten, E. (2014). A more efficient approach to parallel-tempered Markov-chain Monte Carlo for the highly structured posteriors of gravitational-wave signals. Phys. Rev. D, 90(2), 024014. doi: 10.1103/PhysRevD.90.024014

Feroz, F., Gair, J. R., Hobson, M. P., \& Porter, E. K. (2009, oct). Use of the MULTINEST algorithm for gravitational wave data analysis. Classical and Quantum Gravity, 26(21), 215003. Retrieved from https://doi.org/10.1088\%2F0264-9381\% 2F 262F 212F215003 doi: 10.1088/0264-9381/26/21/215003

Feroz, F., \& Skilling, J. (2013). Exploring multi-modal distributions with nested sampling. In Aip conference proceedings. (Vol. 1553, pp. 106-113). New York :: American Institute of Physics.

Finn, L. S. (1992). Detection, measurement and gravitational radiation. arXiv.org, 46(12). Retrieved from http://search.proquest.com/docview/2090129784/

Fishbach, M., Holz, D., \& Farr, W. (2018). Does the black hole merger rate evolve with redshift? arXiv.org, 863(2). Retrieved from http://search.proquest.com/ docview/2073588190/

Foreman-Mackey, D., Hogg, D. W., Lang, D., \& Goodman, J. (2013). : The mcmc hammer. Publications of the Astronomical Society of the Pacific., 125(925), 306-312.

Gabbard, H., Messenger, C., Heng, I. S., Tonolini, F., \& Murray-Smith, R. (2019). Bayesian parameter estimation using conditional variational autoencoders for gravitational- 
wave astronomy. arXiv.org. Retrieved from http://search.proquest.com/ docview/2291101509/

Gabbard, H., Williams, M., Hayes, F., \& Messenger, C. (2018). Matching matched filtering with deep networks for gravitational-wave astronomy. Physical review letters., 120(14).

Gair, J. R., Feroz, F., Babak, S., Graff, P., Hobson, M. P., Petiteau, A., \& Porter, E. K. (2010, may). Nested sampling as a tool for LISA data analysis. Journal of Physics: Conference Series, 228, 012010. Retrieved from https://doi.org/10.1088\% 2F1742-65962F2282F12F012010 doi: 10.1088/1742-6596/228/1/012010

Gair, J. R., \& Porter, E. K. (2009, oct). Cosmic swarms: a search for supermassive black holes in the LISA data stream with a hybrid evolutionary algorithm. Classical and Quantum Gravity, 26(22), 225004. Retrieved from https:// doi . org/10.1088\% 2F0264-93812F262F222F225004 doi: 10.1088/0264-9381/26/22/225004

Gebhard, T., Kilbertus, N., Harry, I., \& Schölkopf, B. (2019). Convolutional neural networks: A magic bullet for gravitational-wave detection? Physical Review D, 100(6). Retrieved from https://www.scopus.com/inward/record .uri?eid=2-s2.0-85073030129\&doi=10.1103\%2fPhysRevD . 100 .063015 \&partner ID $=40$ \& md5 $=4268 \mathrm{c} 5$ ec 7 e 5 f $775 \mathrm{~d} 2591 \mathrm{~cd} 464 \mathrm{c} 6 \mathrm{~b} 21 \mathrm{ce}$ (cited By 6) doi: 10.1103/PhysRevD.100.063015

Gelman, A., Carlin, J. B., Stern, H. S., \& Rubin, D. B. (2014). Bayesian data analysis (Third edition ed.). New York, NY, USA: Chapman \& Hall.

George, D., \& Huerta, E. (2018). Deep learning for real-time gravitational wave detection and parameter estimation: Results with advanced ligo data. Physics letters:, 778, 64-70.

George, D., Shen, H., \& Huerta, E. A. (2018, May). Classification and unsupervised clustering of ligo data with deep transfer learning. Phys. Rev. D, 97, 101501. Retrieved from https://link.aps.org/doi/10.1103/PhysRevD.97.101501 doi: 10.1103/PhysRevD.97.101501

Ghonge, S., Chatziioannou, K., Clark, J., Littenberg, T., Millhouse, M., Cadonati, L., \& Cornish, N. (2020). Reconstructing gravitational wave signals from binary black hole mergers with minimal assumptions. arXiv.org. Retrieved from http://search .proquest.com/docview/2382276963/

Goodfellow, I. (2016). Deep learning.

Gossan, S. E., et al. (2015). Observing Gravitational Waves from Core-Collapse Supernovae. In preparation, LIGO-P1400233.

Green, P. (1995). Reversible jump markov chain monte carlo computation and bayesian model determination. Biometrika, 82(4), 711-732. doi: 10.1093/biomet/82.4.711 
Green, S. R., Simpson, C., \& Gair, J. (2020). Gravitational-wave parameter estimation with autoregressive neural network flows.

Hannam, M., Schmidt, P., Bohé, A., Haegel, L., Husa, S., Ohme, F., .. Pürrer, M. (2014). A simple model of complete precessing black-hole-binary gravitational waveforms. arXiv.org, 113(15). Retrieved from http://search.proquest.com/ docview/2084895697/

Hariharan, P. (2007). Basics of interferometry (2nd ed. ed.). Amsterdam ;: Elsevier Academic Press.

Heng, I. (2009, apr). Rotating stellar core-collapse waveform decomposition: a principal component analysis approach. Classical and Quantum Gravity, 26(10), 105005. Retrieved from https://doi.org/10.1088\%2F0264-9381\%2F26\% 2F102F105005 doi: 10.1088/0264-9381/26/10/105005

Heng, I., \& Messenger, C. (2019). Detection and classification of supernova gravitational waves signals: A deep learning approach. arXiv.org. Retrieved from http://search .proquest.com/docview/2331700621/

Hirata, K., Kajita, T., Koshiba, M., Nakahata, M., Oyama, Y., Sato, N., ... Cortez, B. G. (1987, Apr). Observation of a neutrino burst from the supernova sn1987a. Phys. Rev. Lett., 58, 1490-1493. Retrieved from https://link.aps.org/doi/10.1103/ PhysRevLett. 58.1490 doi: 10.1103/PhysRevLett.58.1490

Hulse, R. A., \& Taylor, J. H. (1975, January). Discovery of a pulsar in a binary system. The Astrophysical Journal Letters, 195, L51-L53. doi: 10.1086/181708

Iess, A., Cuoco, E., Morawski, F., \& Powell, J. (2020). Core-collapse supernova gravitational-wave search and deep learning classification. arXiv:2001.00279 [gr-qc].

Janka, H.-T. (2012). Explosion mechanisms of core-collapse supernovae. Annual Review of Nuclear and Particle Science, 62(1), 407-451. Retrieved from https: / / doi.org/ 10.1146/annurev-nucl-102711-094901 doi: 10.1146/annurev-nucl-102711 $-094901$

Katz, M. L., Marsat, S., Chua, A. J. K., Babak, S., \& Larson, S. L. (2020). Gpu-accelerated massive black hole binary parameter estimation with lisa.

Khan, S., Husa, S., Hannam, M., Ohme, F., Pürrer, M., Jimnez Forteza, X., \& Bohé, A. (2016). Frequency-domain gravitational waves from nonprecessing black-hole binaries. II. A phenomenological model for the advanced detector era. Phys. Rev. D, 93(4), 044007. doi: 10.1103/PhysRevD.93.044007

Kirch, C., Edwards, M. C., Meier, A., \& Meyer, R. (2019). Beyond Whittle: Nonparametric correction of a parametric likelihood with a focus on bayesian time series analysis. Bayesian analysis, 14(4), 1037-1073. 
Krolak, A., \& Schutz, B. (1987). Coalescing binaries-probe of the universe. , 19(12), $1163-1171$.

Kuroda, T., Kotake, K., Hayama, K., \& Takiwaki, T. (2017, dec). Correlated signatures of gravitational-wave and neutrino emission in three-dimensional general-relativistic corecollapse supernova simulations. The Astrophysical Journal, 851(1), 62. Retrieved from https://doi.org/10.384721538-43572aa988d doi: 10.3847/1538 $-4357 / \mathrm{aa} 988 \mathrm{~d}$

Lange, J., O'Shaughnessy, R., \& Rizzo, M. (2018). Rapid and accurate parameter inference for coalescing, precessing compact binaries.

LIGO Scientific Collaboration. (2018). LIGO Algorithm Library - LALSuite. free software (GPL). Retrieved from https://lscsoft.docs.ligo.org/lalsuite/ lalsimulation/index.html doi: 10.7935/GT1W-FZ16

LIGO Scientific Collaboration, Virgo Collaboration. (2017).

GRB Coordinates Network, 21513. Retrieved from https://gcn.gsfc.nasa . $g \circ v / g c n 3 / 21513 \cdot g c n 3$

LIGO Scientific Collaboration, Virgo Collaboration. (2020a). GraceDB - GravitationalWave Candidate Event Database. Retrieved from https: / / gracedb. Iigo.org/ superevents/public/03/

LIGO Scientific Collaboration, Virgo Collaboration. (2020b). LVC Software at GWOSC. Retrieved from https://www.gw-openscience.org/software/

Littenberg, T., Cornish, N., Lackeos, K., \& Robson, T. (2020, 4). Global Analysis of the Gravitational Wave Signal from Galactic Binaries.

Littenberg, T. B., \& Cornish, N. J. (2009, Sep). Bayesian approach to the detection problem in gravitational wave astronomy. Phys. Rev. D, 80, 063007. Retrieved from https://link.aps.org/doi/10.1103/PhysRevD.80.063007 doi: 10.1103/PhysRevD.80.063007

Littenberg, T. B., \& Cornish, N. J. (2015, Apr). Bayesian inference for spectral estimation of gravitational wave detector noise. Physical Review D, 91(8). Retrieved from http://dx.doi.org/10.1103/PhysRevD.91.084034 doi: 10.1103/ physrevd.91.084034

Littenberg, T. B., Coughlin, M., Farr, B., \& Farr, W. (2013). Fortifying the characterization of binary mergers in ligo data. arXiv.org, 88(8). Retrieved from http://search .proquest.com/docview/2085920487/

Logue, J., Ott, C. D., Heng, I., Kalmus, P., \& Scargill, J. H. C. (2012, Aug). Inferring corecollapse supernova physics with gravitational waves. Phys. Rev. D, 86, 044023. Retrieved from https://link.aps.org/doi/10.1103/PhysRevD.86.044023 doi: 10.1103/PhysRevD.86.044023 
Lynch, R., Vitale, S., Essick, R., Katsavounidis, E., \& Robinet, F. (2017). Informationtheoretic approach to the gravitational-wave burst detection problem. Phys. Rev., D95(10), 104046. doi: 10.1103/PhysRevD.95.104046

Maggiore, M., Broeck, C. V. D., Bartolo, N., Belgacem, E., Bertacca, D., Bizouard, M. A., ... Sakellariadou, M. (2020). Science case for the einstein telescope. Journal of Cosmology and Astroparticle Physics, 2020(03), 050-050.

Mandic, V., Thrane, E., Giampanis, S., \& Regimbau, T. (2012, Oct). Parameter estimation in searches for the stochastic gravitational-wave background. Phys. Rev. Lett., 109, 171102. Retrieved from https://link.aps.org/doi/10.1103/ PhysRevLett.109.171102 doi: 10.1103/PhysRevLett.109.171102

Marsat, S., Baker, J. G., \& Canton, T. D. (2020). Exploring the bayesian parameter estimation of binary black holes with lisa.

Maturana-Russel, P., Brewer, B. J., Klaere, S., \& Bouckaert, R. R. (2019, 06). Model Selection and Parameter Inference in Phylogenetics Using Nested Sampling. Systematic Biology, 68(2), 219-233. Retrieved from https://doi.org/10.1093/sysbio/ syy0 50 doi: $10.1093 /$ sysbio/syy050

Maturana-Russel, P., \& Meyer, R. (2019). Bayesian spectral density estimation using psplines with quantile-based knot placment. arXiv.org. Retrieved from http: / / search .proquest.com/docview/2221135074/

Maturana-Russel, P., \& Meyer, R. (2020). psplinepsd: P-splines for spectral density estimation [Computer software manual]. Retrieved from https://github.com/ pmat 747/psplinePsd

Maturana-Russel, P., Meyer, R., Veitch, J., \& Christensen, N. (2019, Apr). Stepping-stone sampling algorithm for calculating the evidence of gravitational wave models. Phys. Rev. D, 99, 084006. Retrieved from https://link.aps.org/doi/10.1103/ PhysRevD.99.084006 doi: 10.1103/PhysRevD.99.084006

Meier, A., Kirch, C., Edwards, M. C., Meyer, R., \& Christensen, N. (2018). beyondwhittle: Bayesian spectral inference for stationary time series [Computer software manual]. Retrieved from https://CRAN.R-project.org/package=beyondWhittle ( $\mathrm{R}$ package version 0.6.0)

Metropolis, N., Rosenbluth, A., Rosenbluth, M., Teller, A., \& Teller, E. (1953). Equation of state calculations by fast computing machines. The Journal of Chemical Physics, 21(6), 1087-1092.

Meyer, R., \& Christensen, N. (2016). Gravitational waves: A statistical autopsy of a black hole merger. Significance, 13(2), 20-25. Retrieved from https://rss.onlinelibrary.wiley.com/doi/abs/10.1111/ j.1740-9713.2016.00896.x doi: 10.1111/j.1740-9713.2016.00896.x 
Nagar, A., et al. (2018). Time-domain effective-one-body gravitational waveforms for coalescing compact binaries with nonprecessing spins, tides and self-spin effects. Phys. Rev. D, 98(10), 104052. doi: 10.1103/PhysRevD.98.104052

Nuttall, L. (2018). Characterizing transient noise in the ligo detectors. Philosophical Transactions. Series A, Mathematical, Physical, and Engineering Sciences, 376(2120), 20170286-20170286. Retrieved from http://search.proquest .com/docview/2025262304/

Ossokine, S., Buonanno, A., Marsat, S., Cotesta, R., Dietrich, T., Haas, R., .. Szilágyi, B. (2020). Multipolar effective-one-body waveforms for precessing binary black holes: Construction and validation. arXiv.org. Retrieved from http: / / search. proquest .com/docview/2392836557/

Ott, C. D., Burrows, A., Livne, E., \& Walder, R. (2004). Gravitational waves from axisymmetric rotating stellar core collapse. The Astrophysical Journal, 600, 834-867.

Pai, A., Dhurandhar, S., \& Bose, S. (2001). Data-analysis strategy for detecting gravitational-wave signals from inspiraling compact binaries with a network of laserinterferometric detectors. Physical Review D - Particles, Fields, Gravitation and Cosmology, 64(4), 30. doi: 10.1103/PhysRevD.64.042004

Pankow, C., Brady, P., Ochsner, E., \& O’Shaughnessy, R. (2015, Jul). Novel scheme for rapid parallel parameter estimation of gravitational waves from compact binary coalescences. Phys. Rev. D, 92, 023002. Retrieved from https:// link.aps.org/doi/ 10.1103/PhysRevD.92.023002 doi: 10.1103/PhysRevD.92.023002

Pitkin, M., Gill, C., Veitch, J., Macdonald, E., \& Woan, G. (2012). A new code for parameter estimation in searches for gravitational waves from known pulsars. Journal of physics., 363(1), 012041.

Pitkin, M., Isi, M., Veitch, J., \& Woan, G. (2017). A nested sampling code for targeted searches for continuous gravitational waves from pulsars. arXiv.org. Retrieved from http://search.proquest.com/docview/2075868092/

Pitkin, M., Reid, S., Rowan, S., \& Hough, J. (2011). Gravitational wave detection by interferometry (ground and space). Living Reviews in Relativity, 14, 5. Retrieved from https://doaj.org/article/e647eb6f49194e84b57d0d422665d916

Powell, J., Gossan, S. E., Logue, J., \& Heng, I. S. (2016, Dec). Inferring the corecollapse supernova explosion mechanism with gravitational waves. Phys. Rev. D, 94, 123012. Retrieved from https://link.aps.org/doi/10.1103/PhysRevD .94 .123012 doi: 10.1103/PhysRevD.94.123012

Powell, J., Szczepanczyk, M., \& Heng, I. S. (2017, Dec). Inferring the core-collapse supernova explosion mechanism with three-dimensional gravitational-wave simulations. Phys. 
Rev. D, 96, 123013. Retrieved from https://link.aps.org/doi/10.1103/ PhysRevD.96.123013 doi: 10.1103/PhysRevD.96.123013

Pratten, G., Husa, S., Garcia-Quiros, C., Colleoni, M., Ramos-Buades, A., \& Estelles, H. (2020). Setting the cornerstone for the imrphenomx family of models for gravitational waves from compact binaries: The dominant harmonic for non-precessing quasicircular black holes. arXiv.org. Retrieved from http: // search.proquest.com/ docview/2349068550/

LIGO Scientific, \& Virgo Collaborations. (2017). The basic physics of the binary black hole merger gw150914. Annalen der Physik, 529(1-2). Retrieved from https://www. scopus. com/inward/record.uri?eid=2-s2 $.0-84990230991 \& d o i=10.1002 \% 2$ fandp $.201600209 \&$ partner $I D=$ $40 \& \mathrm{md} 5=4$ eeff6b1c4e97747fba579ae62d25910 (cited By 31) doi: 10.1002/andp. 201600209

Pürrer, M. (2015). Frequency domain reduced order model of aligned-spin effective-onebody waveforms with generic mass-ratios and spins. arXiv.org, 93(6). Retrieved from http: //search.proquest.com/docview/2077177660/

Pürrer, M. (2016). Frequency domain reduced order model of aligned-spin effectiveone-body waveforms with generic mass ratios and spins. Physical Review D, 93(6). Retrieved from https://www.scopus.com/inward/record .uri?eid=2-s2.0-84962682539\&doi=10.1103\%2 fPhysRevD . 93 .064041 \&partner ID $=40$ \& md5 = 76 fe $31 \mathrm{~cd} 88 \mathrm{aca} 8293005 \mathrm{cad} 55 \mathrm{cc} 48 \mathrm{f} 11$ doi: 10.1103/PhysRevD.93.064041

Robson, T., \& Cornish, N. (2017, nov). Impact of galactic foreground characterization on a global analysis for the LISA gravitational wave observatory. Classical and Quantum Gravity, 34(24), 244002. Retrieved from https://doi.org/10.1088\%2F1361 $-6382 \div 2 \mathrm{Faa} 9601$ doi: 10.1088/1361-6382/aa9601

Roma, V., Powell, J., Heng, I., \& Frey, R. (2019). Astrophysics with core-collapse supernova gravitational wave signals in the next generation of gravitational wave detectors. arXiv.org. Retrieved from http: //search.proquest.com/docview/ $2171891046 /$

Romano, J. D., \& Cornish, N. J. (2017). Detection methods for stochastic gravitationalwave backgrounds: a unified treatment. Living Rev. Rel., 20(1), 2. doi: 10.1007/s41114 $-017-0004-1$

Romero-Shaw, I., Talbot, C., Biscoveanu, S., D’Emilio, V., Ashton, G., Berry, C., ... Xiao, L. (2020). Bayesian inference for compact binary coalescences with bilby: Validation and application to the first ligo-virgo gravitational-wave transient catalogue. arXiv.org. Retrieved from http://search.proquest.com/docview/2408963264/

Röver, C., Bizouard, M.-A., Christensen, N., Dimmelmeier, H., Heng, I., \& Meyer, R. 
(2009). Bayesian reconstruction of gravitational wave burst signals from simulations of rotating stellar core collapse and bounce. Physical Review D - Particles, Fields, Gravitation and Cosmology, 80(10). doi: 10.1103/PhysRevD.80.102004

Röver, C., Meyer, R., \& Christensen, N. (2006). Bayesian inference on compact binary inspiral gravitational radiation signals in interferometric data. Class. Quant. Grav., 23, 4895-4906. doi: 10.1088/0264-9381/23/15/009

Röver, C., Meyer, R., \& Christensen, N. (2007). Coherent bayesian inference on compact binary inspirals using a network of interferometric gravitational wave detectors. Physical Review D - Particles, Fields, Gravitation and Cosmology, 75(6). doi: 10.1103/PhysRevD .75 .062004

Röver, C., Meyer, R., \& Christensen, N. (2011). Modelling coloured residual noise in gravitational-wave signal processing. Classical and Quantum Gravity, 28(1), 015010.

Sachdev, S., Regimbau, T., \& Sathyaprakash, B. (2020). Subtracting compact binary foreground sources to reveal primordial gravitational-wave backgrounds. arXiv.org. Retrieved from http://search.proquest.com/docview/2355121671/

Sathyaprakash, B., Abernathy, M., Acernese, F., Ajith, P., Allen, B., Amaro-Seoane, P., ... Bizouard, M. (2012). Scientific objectives of einstein telescope. Classical and Quantum Gravity.

Schutz, B. F. (2009). A first course in general relativity (Second edition.. ed.).

Setyawati, Y., Pürrer, M., \& Ohme, F. (2020, mar). Regression methods in waveform modeling: a comparative study. Classical and Quantum Gravity, 37(7), 075012. Retrieved from https://doi.org/10.1088\%2F1361-6382\%2Fab693b doi: 10.1088/1361-6382/ab693b

Shen, H., Huerta, E., Zhao, Z., Jennings, E., \& Sharma, H. (2019). Deterministic and bayesian neural networks for low-latency gravitational wave parameter estimation of binary black hole mergers. arXiv.org. Retrieved from http://search.proquest .com/docview/2188818633/

Singer, L., Chen, H., Holz, D., Farr, W., Price, L., Raymond, V., ... Mandel, I. (2016). Going the distance: Mapping host galaxies of ligo and virgo sources in three dimensions using local cosmography and targeted follow-up. Astrophysical Journal Letters, 829, $1-7$.

Singer, L. P., Chen, H.-Y., Holz, D. E., Farr, W. M., Price, L. R., Raymond, V., ... Mandel, I. (2016). Supplement: "going the distance: mapping host galaxies of ligo and virgo sources in three dimensions using local cosmography and targeted follow-up" ( 2016, apjl, 829, 115 ). The Astrophysical Journal Supplement Series, 226(1), 10.

Singer, L. P., \& Price, L. R. (2016, Jan). Rapid Bayesian position reconstruction for gravitational-wave transients. Phys. Rev. D, 93, 024013. Retrieved from 
https://link.aps.org/doi/10.1103/PhysRevD.93.024013 doi: 10 .1103/PhysRevD.93.024013

Skilling, J. (2006, 12). Nested sampling for general bayesian computation. Bayesian Anal., 1(4), 833-859. Retrieved from https://doi.org/10.1214/06-BA127 doi: 10.1214/06-BA127

Smith, R., \& Ashton, G. (2019, 9). Expediting Astrophysical Discovery with Gravitational-Wave Transients Through Massively Parallel Nested Sampling. arXiv eprints, arXiv:1909.11873.

Smith, R., Field, S. E., Blackburn, K., Haster, C.-J., Pürrer, M., Raymond, V., \& Schmidt, P. (2016, Aug). Fast and accurate inference on gravitational waves from precessing compact binaries. Phys. Rev. D, 94, 044031. Retrieved from https : / / ink . aps . org/doi/ 10.1103/PhysRevD.94.044031 doi: 10.1103/PhysRevD.94.044031

Smith, R., Talbot, C., Vivanco, F. H., \& Thrane, E. (2020). Inferring the population properties of binary black holes from unresolved gravitational waves.

Smith, T. L., \& Caldwell, R. (2019). LISA for Cosmologists: Calculating the Signal-toNoise Ratio for Stochastic and Deterministic Sources. Phys. Rev. D, 100(10), 104055. doi: 10.1103/PhysRevD.100.104055

Somiya, K. (2012). Detector configuration of kagra-the japanese cryogenic gravitationalwave detector. Classical and Quantum Gravity, 29(12), 124007.

Stevenson, S., Berry, C. P. L., \& Mandel, I. (2017). Hierarchical analysis of gravitationalwave measurements of binary black hole spin-orbit misalignments. Monthly Notices of the Royal Astronomical Society, 471(3), 2801-2811.

Stroeer, A., et al. (2007). Inference on white dwarf binary systems using the first round Mock LISA Data Challenges data sets. Class. Quant. Grav., 24, S541-S550. doi: 10 .1088/0264-9381/24/19/S17

Stuver, A. (2020). LIGO Scientific Collaboration Astrophysical Sources. Retrieved from https://www.ligo.org/multimedia/gallery/ast.php

Summerscales, T. Z., Burrows, A., Finn, L. S., \& Ott, C. D. (2008, may). Maximum entropy for gravitational wave data analysis: Inferring the physical parameters of corecollapse supernovae. The Astrophysical Journal, 678(2), 1142-1157. Retrieved from https://doi.org/10.1086\%2F528362 doi: 10.1086/528362

Sun, L., Goetz, E., Kissel, J., Betzwieser, J., Karki, S., Viets, A., .. Urban, A. (2020). Characterization of systematic error in advanced ligo calibration. arXiv.org. Retrieved from http://search.proquest.com/docview/2399576740/

Swendsen, R. H., \& Wang, J.-S. (1986). Replica monte carlo simulation of spin-glasses. Physical Review Letters, 57(21), 2607-2609. 
Talbot, C., \& Thrane, E. (2020). Gravitational-wave astronomy with an uncertain noise power spectral density. arXiv.org. Retrieved from http://search.proquest .com/docview/2411945589/

Taylor, J. H., \& Weisberg, J. M. (1982, February). A new test of general relativity Gravitational radiation and the binary pulsar PSR 1913+16. Astrophys. J., 253, 908-920. doi: $10.1086 / 159690$

Thrane, E., \& Talbot, C. (2019). An introduction to bayesian inference in gravitational-wave astronomy: Parameter estimation, model selection, and hierarchical models. Publications of the Astronomical Society of Australia, 36, e010. doi: 10.1017/pasa.2019.2

Tinto, M., \& Dhurandhar, S. V. (2014). Time-delay interferometry. Living Reviews in Relativity, 17(1), 6. doi: 10.12942/lrr-2014-6

Toubiana, A., Marsat, S., Babak, S., Baker, J., \& Canton, T. D. (2020). Parameter estimation of stellar-mass black hole binaries with lisa.

Umstätter, R., Christensen, N., Hendry, M., Meyer, R., Simha, V., Veitch, J., ... Woan, G. (2005a, Jul). Bayesian modeling of source confusion in lisa data. Phys. Rev. D, 72, 022001. Retrieved from https://link.aps.org/doi/10.1103/PhysRevD .72 .022001 doi: 10.1103/PhysRevD.72.022001

Umstätter, R., Christensen, N., Hendry, M., Meyer, R., Simha, V., Veitch, J., ... Woan, G. (2005b, aug). LISA source confusion: identification and characterization of signals. Classical and Quantum Gravity, 22(18), S901-S911. doi: 10.1088/0264-9381/22/18/ s04

Umstätter, R., Meyer, R., Dupuis, R. J., Veitch, J., Woan, G., \& Christensen, N. (2004, sep). Estimating the parameters of gravitational waves from neutron stars using an adaptive MCMC method. Classical and Quantum Gravity, 21(20), S1655-S1665. doi: 10.1088/ 0264-9381/21/20/008

Unnikrishnan, C. S. (2013). IndIGO and LIGO-India: Scope and plans for gravitational wave research and precision metrology in India. Int. J. Mod. Phys. D, 22, 1341010. doi: $10.1142 / \mathrm{S} 0218271813410101$

van der Sluys, M., Raymond, V., Mandel, I., Röver, C., Christensen, N., Kalogera, V., ... Vecchio, A. (2008). Parameter estimation of spinning binary inspirals using Markovchain Monte Carlo. Class. Quant. Grav., 25, 184011. doi: 10.1088/0264-9381/25/18/ 184011

van der Sluys, M., Röver, C., Stroeer, A., Christensen, N., Kalogera, V., Meyer, R., \& Vecchio, A. (2008). Gravitational-Wave Astronomy with Inspiral Signals of Spinning Compact-Object Binaries. Astrophys. J., 688, L61. doi: 10.1086/595279

Varma, V., Field, S., Scheel, M., Blackman, J., Kidder, L., \& Pfeiffer, H. (2019). Surrogate 
model of hybridized numerical relativity binary black hole waveforms. arXiv.org, 99(6). Retrieved from http://search.proquest.com/docview/2159024976/

Veitch, J., et al. (2015). Parameter estimation for compact binaries with ground-based gravitational-wave observations using the LALInference software library. Phys. Rev. D, 91(4), 042003. doi: 10.1103/PhysRevD.91.042003

Veitch, J., \& Vecchio, A. (2008a, sep). Assigning confidence to inspiral gravitational wave candidates with bayesian model selection. Classical and Quantum Gravity, 25(18), 184010. Retrieved from https://doi.org/10.1088\%2F0264-9381\%2F 25\% 2F182F184010 doi: 10.1088/0264-9381/25/18/184010

Veitch, J., \& Vecchio, A. (2008b, Jul). Bayesian approach to the follow-up of candidate gravitational wave signals. Phys. Rev. D, 78, 022001. Retrieved from https:// I ink .aps.org/doi/10.1103/PhysRevD.78.022001 doi: 10.1103/PhysRevD.78 .022001

Veitch, J., \& Vecchio, A. (2010). Bayesian coherent analysis of in-spiral gravitational wave signals with a detector network. Phys. Rev. D, 81, 062003. doi: 10.1103/PhysRevD.81 .062003

Venumadhav, T., Zackay, B., Roulet, J., Dai, L., \& Zaldarriaga, M. (2019). A new search pipeline for compact binary mergers: Results for binary black holes in the first observing run of advanced ligo. arXiv.org, 100(2). Retrieved from http: / / search. proquest .com/docview/2186924097/

Viets, A. D., Wade, M., Urban, A. L., Kandhasamy, S., Betzwieser, J., Brown, D. A., ... Weinstein, A. J. (2018). Reconstructing the calibrated strain signal in the advanced ligo detectors. Classical and Quantum Gravity, 35(9), 095015.

Vinciguerra, S., Veitch, J., \& Mandel, I. (2017, may). Accelerating gravitational wave parameter estimation with multi-band template interpolation. Classical and Quantum Gravity, 34(11), 115006. Retrieved from https://doi.org/10.1088\%2F1361 $-6382 \div 2$ Faa6d44 doi: 10.1088/1361-6382/aa6d44

Vines, J., Flanagan, E. E., \& Hinderer, T. (2011, Apr). Post-1-newtonian tidal effects in the gravitational waveform from binary inspirals. Phys. Rev. D, 83, 084051. Retrieved from https://link.aps.org/doi/10.1103/PhysRevD.83.084051 doi: 10.1103/PhysRevD.83.084051

Vinet, J.-Y. (2013, April). Some basic principles of a "LISA". Comptes Rendus Physique, 14(4). Retrieved from https://hal.archives-ouvertes.fr/hal -01369369 doi: 10.1016/j.crhy.2013.02.002

Vousden, W. D., Farr, W. M., \& Mandel, I. (2015, 11). Dynamic temperature selection for parallel tempering in Markov chain Monte Carlo simulations. Monthly Notices of the 
Royal Astronomical Society, 455(2), 1919-1937. Retrieved from https : / / doi . org/ $10.1093 / \mathrm{mnras} / \mathrm{stv} 2422$ doi: 10.1093/mnras/stv2422

Welch, P. (1967). The use of fast fourier transform for the estimation of power spectra: A method based on time averaging over short, modified periodograms. IEEE transactions on audio and electroacoustics., 15(2), 70-73.

Whittle, P. (1957). Curve and periodogram smoothing. Journal of the Royal Statistical Society: Series B (Methodological), 19(1), 38-47.

Wysocki, D., Lange, J., \& O’Shaughnessy, R. (2019). Reconstructing phenomenological distributions of compact binaries via gravitational wave observations. arXiv.org, 100(4). Retrieved from http://search.proquest.com/docview/2073796836/

Zackay, B., Dai, L., \& Venumadhav, T. (2018). Relative binning and fast likelihood evaluation for gravitational wave parameter estimation. arXiv.org. Retrieved from http://search.proquest.com/docview/2074056895/

Zackay, B., Venumadhav, T., Roulet, J., Dai, L., \& Zaldarriaga, M. (2019). Detecting gravitational waves in data with non-gaussian noise. arXiv.org. Retrieved from http://search.proquest.com/docview/2274152872/

Zevin, M., Coughlin, S., Bahaadini, S., Besler, E., Rohani, N., Allen, S., ... Kalogera, V. (2017, feb). Gravity spy: integrating advanced LIGO detector characterization, machine learning, and citizen science. Classical and Quantum Gravity, 34(6), 064003. Retrieved from https://doi.org/10.1088\%2F1361-6382\%2Faa5cea doi: 10.1088/ 1361-6382/aa5cea 